LA- -9270-MS

DE82 019711

\title{
Systems Concepts for DOE Facilities: Analysis of PF/LASs Data
}

R. C. Bearse*

D. G. Shirk

R. S. Marshall

C. C. Thomas, Jr.

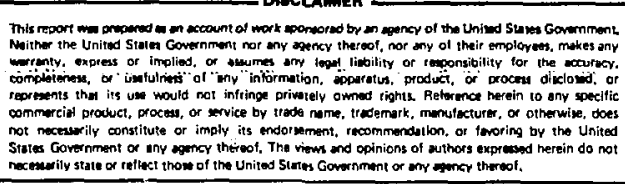

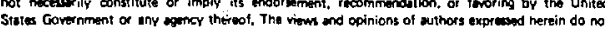

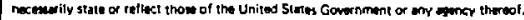

-Vialting Staft Member. Department of Physics and Astronomy, University of Kansas, Lawrence, KS 68045.

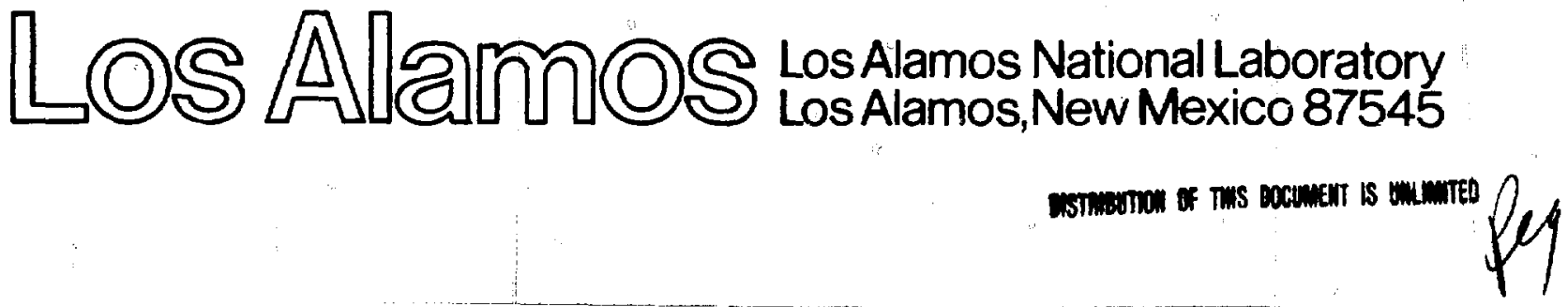




\section{CONTENTS}

ABSTRACT

I. INTRODUCTION

II. BACKGROUND 3

III. THE FFTF PROCESS 4

IV. DATA BASE 6

V. METHODOLOGY

A. Analysis of Measurement Codes 7

B. Materials Balances and MIPs 13

C. Uncertainty Assignments 18

D. Negat1ve Volumes 18

E. Error Propagation , 20

F. Bditing 20

G. Graphical Output 21

H. Types of MIP Charts 21

VI. DISCUSSION $\quad 21$

A. MIP Charts 21

B. CusuM Charts 23

vII. CONCLUSION 26

$\begin{array}{ll}\text { REESRENCES } & 27\end{array}$

APP ENDIX A: TRANSLATION OF DATA 44

$\begin{array}{ll}\text { APP ANDIX B: } & \begin{array}{l}\text { DERTVATION OF THE ERROR } \\ \text { PROPAGATION EQUATION }\end{array}\end{array}$

APPENDIX C: GANLRATION OF MIPs and CUSUMs 


\title{
SYSTEMS CONCEPTS FOR DOE FACILITIES: \\ ANALYSIS OF PF/LASS DATA
}

by

R. C. Bearse, D. G. Shirk, R. S. Marshall, and C. C. Thomas, Jr.

\begin{abstract}
We have analyzed P.lutonium Facility/Los Alamos Safeguards System (PF/LisS) data for the Fast Flux Test Facility (FFTF) process. Highlights of the work are

- the PF/LAss data base provides useful information for accouritability purposes,

- some measurement code assignments appear to be in error,

- some other data are erroneous, and

- material in process (MIP) and cumulative sum (CUSUM) charts are powerful indicators of trouble areas.

From these studies we recommend

- re-examination of instrument biases,

- adoption of new naming procedures for collection batches,

- improvement of measurement code assignment reliability,

- revision of round-off procedures, and

- strengthening of measurement control procedures.
\end{abstract}

\section{INTRODUCTION}

We have analyzed a portion of the Plutonium Facility/Los Alamos Safeguards System (PF/LASS) transaction data base for the operating year February 1980 to February 1981--from cleanout to cleanout. This analysis points up some strengths and weaknesses of the PF/LASS implementation of dynamic accounting (DYMAC) concepts and suggests some 
areas for improvement. The study also suggests ways for buttressing current DYAC principles before implementing similar systems at other operating facilities.

The DYMAC system (PF/LASS) at the Los Alamos National Laboratory Plutonium Processing Facility (TA-55) has been in operation for more than 3 years. PF/LAss has been operated by process staff with.only consultative input from the developers for more than 1 year. Because of the pressures of increasing data flow and requests for changes in the operating system, the operating staff has not had time to analyze PF/IASS data in depth. Moreover, although the coperational safeguards groups and the chemical processing group do have a vested interest in analysts of the data, their emphasis is necessarily on the questions of immediate concern and not on the research and development concern of the efficacy of the system concept. Thus, the Safeguard Systems Group (Q-4) undertook to analyze PF/IASS with attention to its strengths and weaknesses and how it might be improved.

This report underscores the importance of an active research and development role at facilities such as TA-55 for IOS Alamos safeguards research personnel. Conceptual designs, simulations, and off-line demonstrations of nondestructive assay (NDA) equipment are important to the development of effective safeguards, but without extensive experience of in-plät operation such efforts may contain undetected weaknesses that might undermine an otherwise excellent project.

This study will provide useful information to those now responsible for PF/LASS operations. Many of these individuals have been of invaluable assistance in the work reported here...

By its nature our study points primarily to weaknesses in the current system and its mode of operation. We cannot overemphasize, however, that the PF/Lass system is the best of its kind. It works exceedingly well considering its complexity and its nativity as an research and development project. That it works so well is less a tribute to 1ts designers and bullders than to those who, day in and day out, work with it intelligently and with good will. 


\section{BACKGROUND}

This analysis presumes a basic understanding of DMac principles and, more specifically, the operation of PF/LAss. Th1s background is provided but is not exhaustive. For a more detailed account, see Ref. 1 .

TA-55 is batch oriented, that is, special nuclear material (SNM) at TA-55 moves through the plant in identifiable batches. The batches may be divided or combined with other batches to form new ones and may change chemical or physical form. At any time, however, an identifying name is associated with each batch.

The plant has been logically divided into several materials balance areas (MBA) and within these MBAs are subdivisions called receipt areas (RA) or unit processes. The assignment of MBAs and RAs is such that

(I) there is no overlap between RAs,

(2) there is no overlap between MBAs, and

(3) an $R A$ is entirely within a single MBA.

Further, material must, at any time, be completely within one and only one RA (or it must be specifically and identifiably in transit between one RA and another; in-transit items are handied separately from other items, but "in-transit" is logically equivalent to a receipt area within a unique MBA).

NDA instruments are placed strategically throughout the plant so that measurements can be made with certified and calibrated instruments when batches complete key points in their processing, for example, when batches finish a chemical processing step or when batches are aivided or combined.

Before the instruments were constructed and installed, the processing steps were analyzed in detail and protocols were developed for when and how measurements are to be taken. These points were selected because of their importance in assuring adequate accountability and processing information.

Thus, whenever a batch changes form or is sent from one receipt area to another, a "transaction" is made on a terminal linked to a 
central computer, in this case a Data General Eclipse C330. The information contained in this transaction is displayed in Table I. In essence the transaction contains information about the batch location and state before and after alteration or movement as well as the amount of material involved in the transfer. There is also room for comments by the operator or management.

The measurement code and the uncertainty fields associated with each transaction are of particular interest to our analysis. The measurement code is a 3-character code associated with a specific measurement or analysis. Because detailed records are maintained on the precision and accuracy of each instrument as part of the measurement control procedures, the measurement code should allow us to associate random and systematic error variances with each measurement. The uncertainty fields are not now used by PF/LASS, but they reserve space for future use. The assignment of uncertainties will be discussed later.

It should be emphasized that measurements need not always be performed. There are cases where the protocol allows estimation or inference of the sin content. When estimation occurs, special measurement codes are used to signal this fact. Also, PF/LASs programming, apparently, retains the last used measurement code with a batch until it is changed. Thus, a measurement code need not necessarily imply a measurement. As we will point out, this is a weakness in the system that can be easily corrected. Protocol does denand that whenever a batch changes name or receipt area it must be measured.

The PF/LAss computer accepts transactions for a month or more and then, because of size limitations, must transfer this information to off-line bulk storage. It is this bulk-stored data that we analyzed.

III. THE EFTF PROCESS

Our analysis is restricted to the East Flux Test Facility (FWF) process. A flow chart of the process, adapted from Ref. 2, is ahown in Fig. 2. This process ws selected as exemplary of PF/Has data, not only because of its representative nature, but because it has been 
TABLE I

DYMAC TRANSACTION STRUCTURE

- Word No.

$$
1
$$

2,3

4

5-9

10

11,12

13-17

18,19

20,21

22

23,24

25-49

50-51

51-101

102-104

105-107

108-110

111,112

113,114

115, 116

117,118

119-142

143 .

144

145-148

149,150

151,152

153

154, 155

156,157

158

159

160,161

162,163

164,165
Content

Transaction type

MBA

Material type

LOT ID number

Receipt area

Project

Person

Location

Shelf

Special designator

Item description

Remarks

Destination

FFOM INFORMATION

Transaction ID

Date

Time

SNM amount

Uncertainty, SNM amount

Enrichment

Uncertainty, enrichment

Isotope breakdown

Number of impurities

COEI number

seal number

Heasurement code

Bulk amount

Bulk units

Verification amount

Verification instrument

Edited measurement code ${ }^{a}$

Reserved for tagging

Total variance ${ }^{a}$

Random variance

Systematic variance ${ }^{a}$
Format

Integer

Alpha

Alpha

Alpha

Alpha

Alpha

Alpha

Alpha

Alpha

Alpha

Alpha

Alpha

Alpha

Same as To format

Alpha

Integer

Integer

Real

Real

Real

Real

Real

Integer

Integer

Alpha

Alpha

Real

Alpha.

Real (Used for external

Alpha transactions on $l_{y}$ )

Alpha

Alpha

Real

Real

Real

Does not appear in transaction as generated but is added during off-line analysis. 
thoroughly analyzed by the Safeguards Systems Group personnel and Is relatively well understood. See Ref. 2 for accountability aspects of the FFTF process.

Basically, plutonium metal moves from the vault to button oxidation (BU). The oxide is sent for dissolution (OD) and then on to precipitation and redissolution (PR). This second solution is precipitated to a wet oxalate cake (OY), which is hydrocalcined (HC) and finally moves on to sieving, grinding, blending, and canning (BL). Final canning (FC) follows before return to the vault. In addition to each main product stream, there are SNM side streams feeding small scrap or recycling streams.

IV. DATA BASE

We secured copies of the transaction higtory tapes for July 1979 through March 1981. We have selected for analysis those data from cleanout to cleanout--February 5, 1980, through February 13, 1981. Because some measurements from wet chemical analysis take several months to complete and record, we worked with a data base from a wider time range but focussed on the material in process (MIPs) generated only during the 1 -year period.

These history tapes are written in a special "Data General Dump Format," which is not Immediately compatible with the computer used In our analysis, a Prime computer. Further complicating the translation process were four different versions of the dump format used In generating the needed tapes. In addition, the internal representations of characters (alphanumeric) and real numbers differ in the two machines and thus, translation was necessary even after the dump structure was deciphered. About 1 man-month went into deciphering these tapes and rendering them readable by the Prime. A discussion of the translation process is in App. $\lambda$.

After each month's eet of transactions was decoded, those transactions relating to the FPTF process were identified by their receipt areas-the seven areas derignated BU, OD, PR, OY, HC, BL, and FC. Because activity other than FWH routinely takes place within these 
receipt areas, they serve to select only FFTF data. It was later discovered that some MIP activity taking place from the vault required further data selection: all 1tems with ID names of the form LAOXXXXXX were included in the data base whether or not they began or ended in the seven FFTF receipt areas. While the base was being built, eight words were added to each transaction record for our own use. The assignment of these words is shown in Table $I$. Thus, each transaction record in our data base contains 165 computer words of information.

After each month's set of FFTF transactions was selected, the set was time-ordered to allow the development of audit trails by the methods outlined in Ref. 3 .

The time-ordered files from each month were then concatenated into a single file containing 13235 records of 165 words each. It is maintained in a disk file on a dedicated disk drive.

\section{METHODOLOGY}

A. Analysis of Measurement Codes

There were several studies of the measurement codes assigned to transactions to gain experience in using the data base and to evaluate its integrity. Although we cannot determine if particular measurements are correct, we can determine whether the measurement instrument claimed in a particular transaction is indeed the correct one.

Table II shows the frequency of use of certain measurement codes. This table is based on the entire set of FFTF transactions and shows "a number of instances where suspicious instruments were reported. If only those measurements are accepted as correct that involve instruments physically located in the FFrF process (the ones marked with an asterisk in the Table), then 20.38 of the assigned measurement codes may be in error. Subsequentiy, programs vere written that among other proceciures, tagged those transactions used to define MIPs generated in the FFTF process. This subset of data, clearly of high importance, was reanalyzed for frequency of measurement code assignment. In this instance, applying the above criteria finds 17.78 of the measurement codes in error (Table III). This suggests that if, indeed, there are 


\section{FREQUENCY OF USE OF MEASUREMENT CODES FOR ALL TRANSACPIONS IN THE FETF DATA BASE}

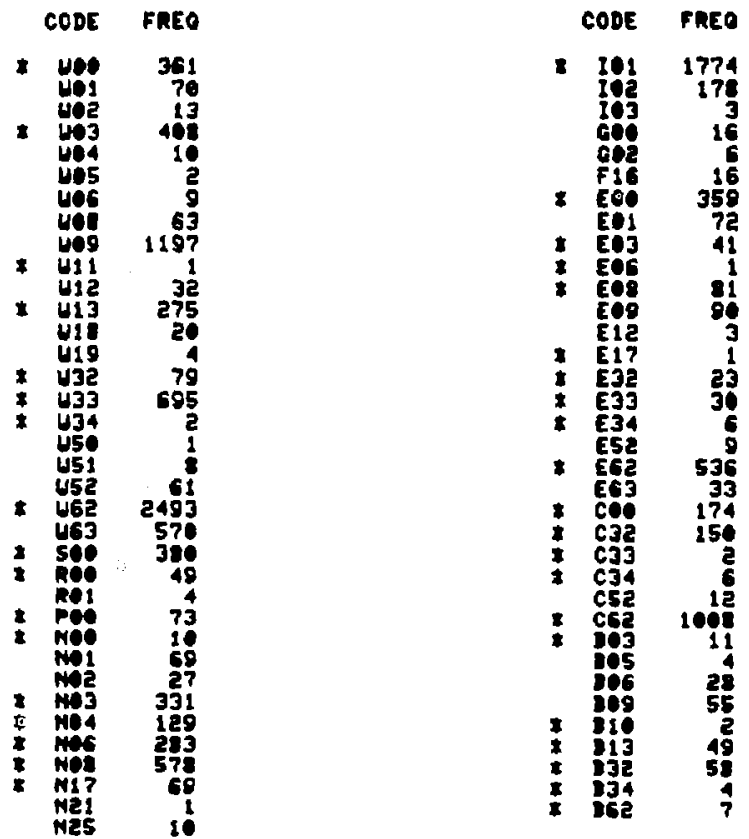

The letter prefix corresponds to the type of instrument or measurement, and the number refers to a particular instrument. The notations are as follows.

$U$ and $B$ - balances

N - thermal neutron counters

I - solution assay instruments

G - segmented gamma scan unit

E - estimated

B. - radiochemical assay

C - west chemistry stochionetry and balance

$P$ - in-process assignment

s - shipper assigned value

mistakes in the measurement code assignments, there are fewer such mistakes among the primary measurements than among the remaining measurements.

These aspersions on measurement code assignmente are not fully fair. Many of these measurements could have been made just as the material was being sent to the FPTF process or imediately after it left. Thus, tranactions involving the FrTF process could have measurement codes associated with other recelpt areas. 
PREQUENCY OF USE OF MEASUREMENT CODES FOR

TRMNSACTIONS USED TO GENERATE MIPS IN THE FETF PROCESS

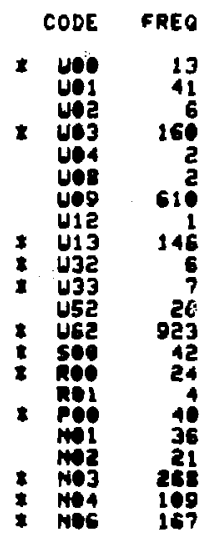

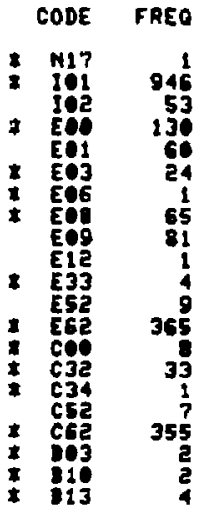

The letter prefix corresponds to the type of instrument or measurement, and the number refers to a particular instrument. The notations are as follows.

$\mathrm{U}$ and $\mathrm{B}$ - balances

N - thernal neutron counters

I - solution assay instruments

G - segnented gamma scan unit

E - estimated

$R$ - radiochemical assay

C - wet chemistry stochimetry and balance

$P$ - in-process assignment

S - shipper assigned value

Since a measurenent code can in principle be assigned to either the "to" side or the "from" side of a transaction, a study was made of the frequency of measurement codes as a function of "to" and "from" location pairs. The results obtained for the full EFTF data base are shown in Table IV.

So that the table would be of manageable length, all BXX, CXX, and FXX measurement codes were changed to wXX. Also, all codes not directly using an instrument and all transactions involving the vault were onitted. 


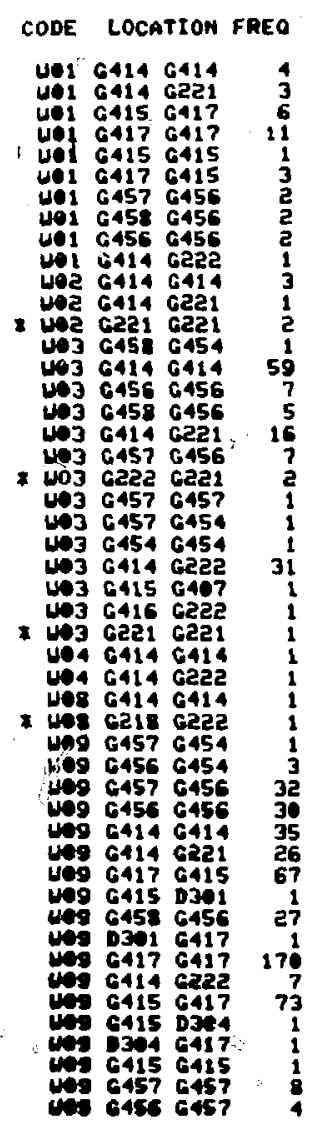

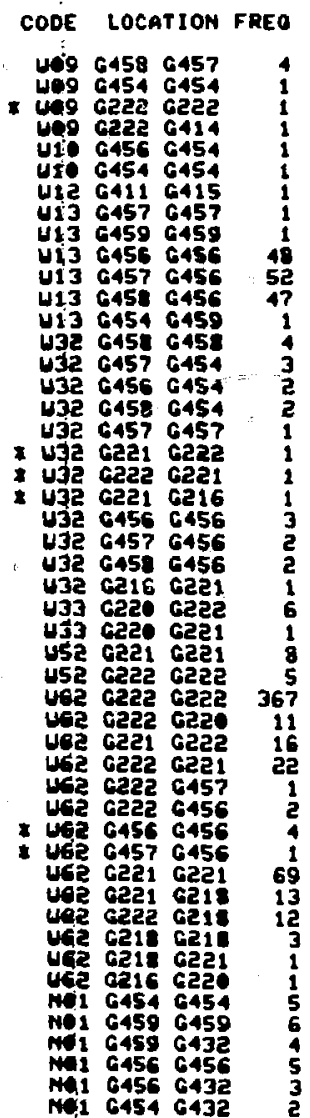

CODE LOCATION FREO

Me1 G415 6415 NOI G415 G440

NeI 6456 G440

- Moz Gezl Geze MoL G222 G445 Ne2 6415 G445 We G456 G456 Me2 64566432 NO2 G414 G414 Me 64146459 Mie 64596459 M. 6456 G45 33 G415 6414 1036414 G414

- No3 G222 Gez2 $N 3$ G222 6432 No3 64596459 No3 G459 6432 Ne3 64146459 Mo3 G4t5 G415 M3 6415 G432

No3 G415 G445

- M3 G22l Ga2t Ne3 G221 G432 Hej 64146445 Mo3 6414 G415 M3 G45 D46 No3 G414 G432 Mo3 G221 G444 - Mo gazl gaza Moj G222 G444 w3 G456 6456 H. 352 G43 Ne3 64546454 Ne3 G454 G444 Ho3 G414 G444 Me3 G414 G415 HO Ge2e G440 No4 6459 6459 Me4 Ga15 G41? Ma Ga1? Ga1? NO4 G415 6415
CODE LOCATION FREO

Ne4 G416 G414 Ne4 G414 G414 Ne4 G414 6416 Nos 6415 6432 NOS G450 6450 Ne6 G459 G432 Ne6 G456 G456 M06 G455 G432 M06 G432 G455 NOS G454 G445 Nes G456 6402 Mes G454 G432 Ne6 G456 G438 - M. Gazo G232 Mivs Ga2e G432 Ho6 G222 G445 $N 06$ G456 G445 N06 G456 6454 NO6 C454 G438 NOS G45? G456 NOS G456 G444 MO6 G454 G440 NOS G454 G444 No6 G45D G440 N06 G459 G444 N06 G456 G440 No6 $6456 \quad 6431$ No6 64596445 N06 G414 6459 Mes G414 G459 mos No G417 6417 Ne8 G454 G45. NoE 64546432 No8 G456 G456 Nos G456 G432 No8 64596432 Hos 64596445 No8 64596440 No8 G415 G414 Nol Ga15 G415 Ne G45? G456 No8 G459 G444
CODE LOCATION FREO

No8 G416 G416 Mas GA16 G444 No8 G416 G414 Me $6417 \mathrm{Ge22}$ Ne 6459 G454 Nol G416 G450 H17 Ga16 Ga1? I01 6414 6414 101 G456 6454 I01 645? 6454 10164586454 10164546455 10164546459 I01 6454 6454 101 To1 6450 G452 I01 6450 G414 1016456 6456 I01 $6454 \mathrm{G} 432$ I01 G456 G452 101 G456 645? I01 6454 G46e I01 $6.456 \quad 6458$ I01 G459 G445 I01 6457 6459 I01 64596454 1016456 G42? I01 G452 G454 I01 G456 G433 101 G454 G45e I01 $6457 \quad 6456$ I01 64546458 10164546456 10264596459 I0e G454 G454 I02 6454 G455 I02 $6454 \mathrm{G459}$ I02 G458 G45 Te G450 G454

The starred entries are those that involve neither a to nor from location in the same wing as the instrument. All transactions involving a vault location on one side of the transaction have been deleted, as have those involving $E$, $S, P, R$, and 00 measurement codes. 
Even with these restrictions, 18 of the codes imply measurements made on instruments not even in the same wing as either the sending or receiving location. These are marked with an asterisk in Table IV. Although there axe not many errors, they lead to some difficulties that will be discussed next. These difficulties also support our contention that there are errors in the data hase.

A comparison of batch names to measurement codes also provides a useful indication of measurement code assignment errors. Batch names are assigned on the basis of the material in the batch and its state of processing. The instruments selected to make a measurement reflect the type of material being measured. For example, it is unlikely that a solid sample of plutonium will be analyzed with the solution assay instrument (SAT) because it was designed for analysis of solutions and can only give meaningful results for solutions. It is reasonable, then, to expect that all samples of similar size and having the same type of batch name be measured with the same type of instrument, if not the same instrument.

The data were analyzed to determine the frequency of measurement code assignments as a function of batch name type. As experted, most of the examples are acceptable and, therefore, are uninteresting (Table V). Some, however, vindicate our contention that there are errors in measurement code assignments. The starred entries show cases where the measurement codes are not consistent with each other. Based on this analysis, we developed procedures, discussed in Sec. V.F., for editing the data base to "correct" these measurement codes.

As will also be discussed below, we developed techniques for calculating MIPs, propagating errors, and plotting the results. Figure 2 shows such a plot for the BU unit process. Note the large error bars on five of the points. (The other error bars are too small to see.) Detailed investigation of these five points indicated that the only peculiar feature of those MIPs was the assignment of an instrument code used at no other time during the year in that receipt area. The large uncertainties associated with the nonstandard instrument led to the large error bars on the plotted point. We believe the measurement code was assigned incorxectly. There are several other, similar, examples in the $\mathrm{BL}, \mathrm{OD}$, and $\mathrm{PR}$ processes. 
FREQUENCY OF MEASUREMENT CODES ASSOCIATED WITH THE PREFIXES OF THE TO AND FROM LOT IDS

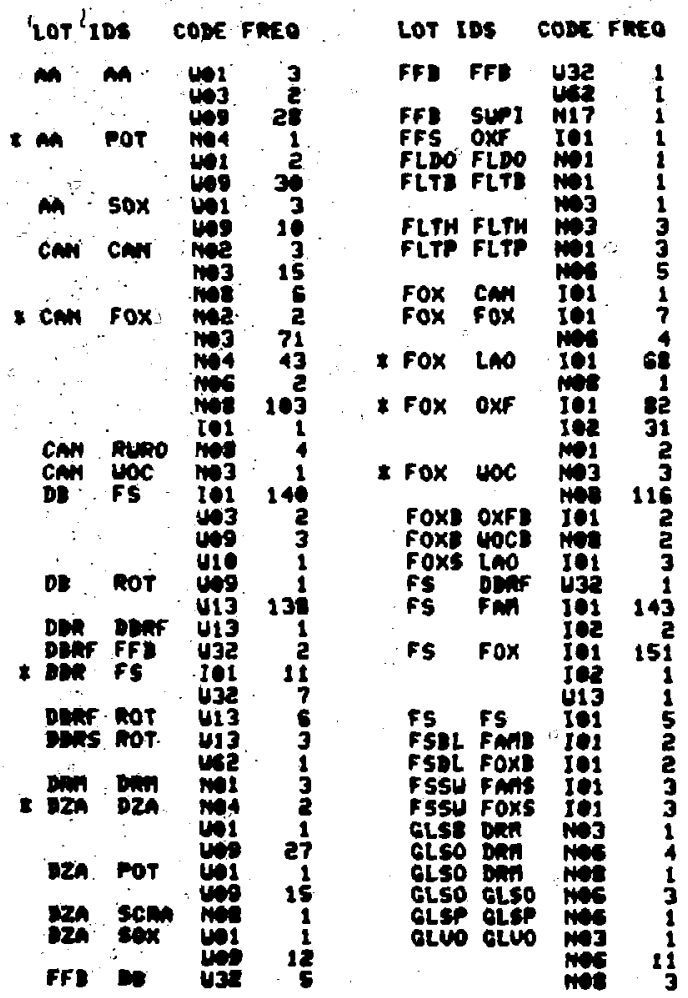
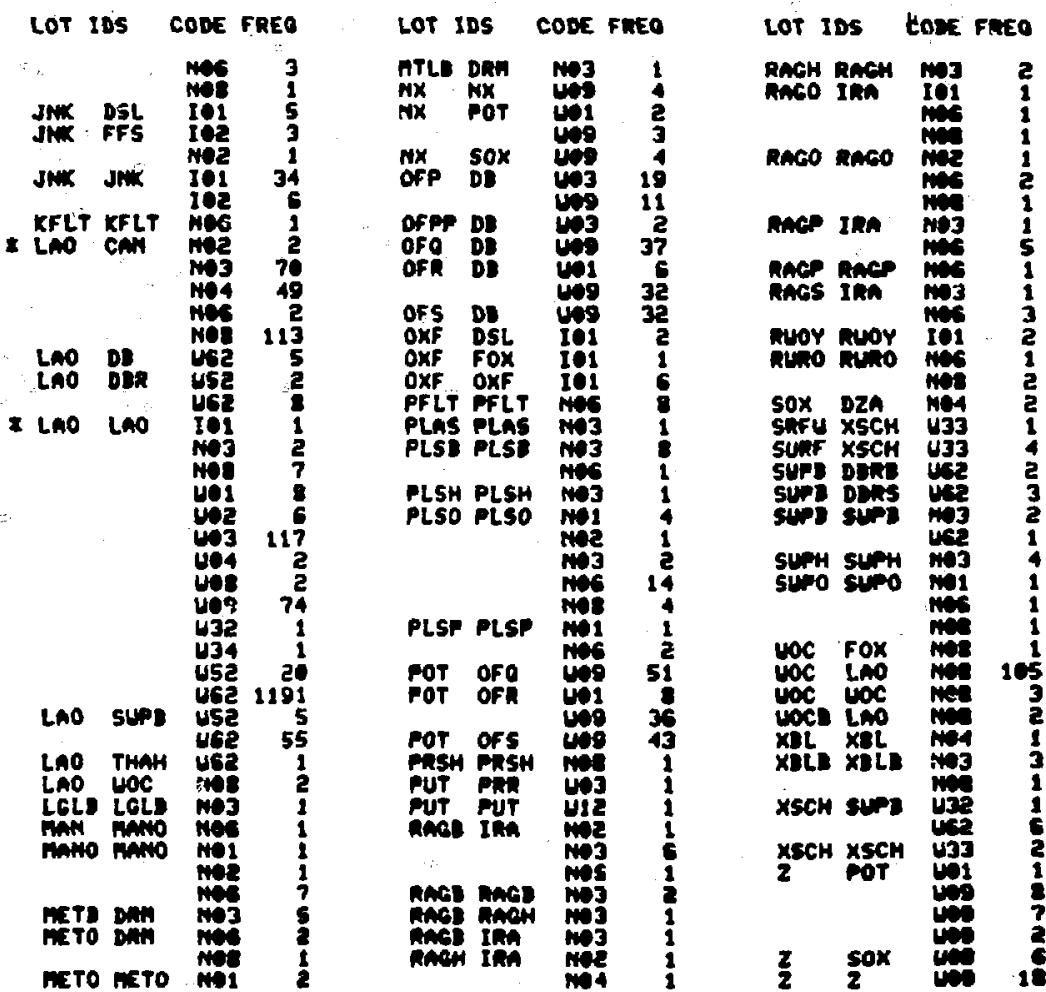

The starred pairs indicate pairs for which measurement codes were edited to the most prevalent code for that Lot ID combination. Again, the codes $E$, $P$, R, and $S$ are excluded and $B$ and $C$ were changed to $w$. 
Although we are hot certain that editing of measurement codes is necessary and are less certain that our editing method is sufficient, the internal evidence appears to warrant and fustify our approach--the resulting data base gives results more in line with expectition.

\section{B. Materials Balances and MIPs}

Two fundamental quantities needed for accounting in the PF/LASS system are the materials balance (MB) and the quantity designated as MIP. A MIP is drawn around batches in a specific receipt area and is obtained by adding material entering a receipt area and subtracting the material leaving on a batch basis. More precisely, for a specified lot $x$,

$$
\operatorname{MIP}_{\mathbf{x}}=\sum_{i=1}^{m} I_{x i}-\sum_{i=1}^{n} 0_{x i}
$$

where

$$
\begin{aligned}
& \text { MIP } x \text { is the MIP for lot } x \text {, } \\
& I_{x i} \text { is the ith item added to the receipt area, } \\
& o_{x i} \text { is the ith item removed from the receipt area, } \\
& m \text { is the number of items added to lot } x \text {, and } \\
& n \text { is the number of items removed from lot } x \text {. }
\end{aligned}
$$

MIP ${ }_{x}$ is the contribution from lot $x$ to the receipt area inventory after lot $x$ processing has been completed. As such it includes equipment and process holdup, all unmeasured side streams as well as, potentially, material unaccounted for (that is, unexplained inventory difference).

In the PF/LASS system, MIP is treated as a nonphysical item of inventory and transactions are made to and from MIP. Specific MIPs accounts are designated as MIPXX where $\mathrm{XX}$ denotes the receipt area involved. Transactions from MIP are generated when plutonium previously transferred to MIP is measured, e.g., as a result of cleanout, or when there is a process gain. 
The MIP Eq. (1) does not include receipt area ending inventory. However, assuming known or zero beginning inventory, the cumulative sum (CUSUM) of the MIPs over a period of time yields the change in book inventory for the receipt area at the end of that time period. If the end of the time period includes a cleanout and physical inventory, the CUSUM 18 the physical inventory of the receipt area.

The materials balance is classically defined as

$$
M B=B I+R-S-E I,
$$

where

BI is the beginning inventory,

$R$ is the receipts during the period,

$S$ is the shipments during the period, and

EI is the ending inventory.

Ideally, the materials balance would be zero, but it is nonzero because of measurement errors, unmeasured side streams, and, potentlally, diversion.

The materials balance can be redefined as

$$
M B=\sum_{i=1}^{m} I_{x i}-\sum_{i=1}^{n} 0_{x i}-E H-P H-M E=M U F
$$

where

$I_{x 1^{\prime}} O_{x 1^{\prime}} m$, and $n$ have the same meaning as in $\mathrm{Eq} .(1)$;

EH 18 the equipment holdup; *

PH is the process holdup; **

\footnotetext{
* Equipment holdup is defined as nuclear material that adherea so tenaclously to the equipent that it has becone part of the equipant or requires special treatmont to remove.

* Process holdup is defined as nuclear material that, although physically inside the process equipant, is part of the flow and is aubject to cleanout.
} 
MF is all forme of masurement, clerical, and other ldentifiable errors

MUF is material unaccounted for (unexplained inventory difference).

Substituting Eq. (1) in Eq. (3) yields

$M B=M I P_{X}-$ FH - PH $-M E=M U F$

Thus, MIP $x$ coupled with measurement or estimation of $\mathrm{EH}, \mathrm{PH}$, and ME provides a materlals balance at completion of the processing of lot $x$ and before initiation of the processing of lot $x+1$.

One primary purpose of our analysis was to verify the values of MIP reported in transactions to MIP accounts. We also sought to identify those transactions associated with each MIP transaction so that uncertainties could be appropriately propagated. Error propagation is necessary because the MIP is inferred, not measured, and thus, the uncertainty in the reported value of MIP can only be determined from the uncertainties of the associated transactions.

There are at least two conceptual procedures for determining the set of transactions associated with a MIP. One procedure uses the audit-trail techniques alscussed in Ref. 3; the other (the batchoriented method) is much simpler and faster to execute with a computer but provides less complete information on the origin of the MIP.

We discuss first the audit-trail approach with reference to Fig. 3. which shows material as it moves into and through a unit process. Each circle represents a discrete stage in the process. The batches start at the top and proceed downward in time. When the batches are shown between the horizontal Iines, we imply they are within the unit process. The vectors joining the circles represent the transaction, and a corresponding transaction record in the computer will indicate the starting and ending points and how much material flowed along the vector. A transfer to MIP is also represented. In the example, two batches, 1 and 2, enter the unit process to be renamed 3 and 4 . They are processed to 5 and 6 and are then combined into $A$. The comblned 
batch 13 subdivided into batches 7 and 8 , which leave the process and become batches 9 and 10. To determine MIP we need only to perform a simple algebraic sum of the vectors that cross the boundaries of the recelpt area--these are shown as broad arrows.

The problem is then two-fold: (1) establish which vectors out of the entire FFTF set are related to a particular MIP transaction and (2) determine which vectors cross the boundary. It is a simple matter to examine the name of the batch that feeds the MIP transaction (in the example it is called $A$ ) and then to determine all other connections from it. Once these connections are determined, the connections to these secondary batches can be identified. The process is repeated until the computer recognizes that it has found all batches within the receipt area. Once the computer has the complete list of transactions leading to the MIP, it can discard any transactions that begin and end within the receipt area (in our example $3+5,4+6, A \rightarrow 7$, and $A \rightarrow B$ ). These are discarded because they do not change the amount of material within the RA. Finally, the remalning transactions can be added and the associated uncertainties propagated to arrive at a final MIP and 1ts assoclated error.

A much less rigorous, but much more rapid, approach was also tried. This approach is consistent with the manner in which the MIP was first generated for Inclusion in the data base. Conceptually, the materials balance is taken around a batch rather than around the receipt area as a whole (Fig. 4). Because MIPs are generated for each batch that enters the receipt area, the result is the same, but information about the detailed steps the material takes through the $R A$ is lost. Although the MIP is defined relative to the receipt area, information about the entrance and, sometimes, exit of the batch $1 \mathrm{~s}$ inferred rather than actual. In Fig. 4 the broad arrows are the transactions included in the sum. Here, the MIP is determined by an algebraic sum of all transactions leading to or from the batch generating the MIP. information about transactions before transactions 5 and 6 is lost, as is information about transactions subsequent to batches 7 and 8. This causes no difficulty because the operating rules at TA-55 require that any change in a batch subequent to entrance or before exit would require generation of a separate MIP. We found no cases 
where differences between our calculations of MIP and the recorded value can be ascribed to this algorithm. (There are two cases where a 2-g difference occurs between our calculation and the recorded one, but both involve large batches and many transactions and can be adequately explained by our having left some needed transactions out of our data base. During the developmenc of this analysis such discrepancies often arose and, in each case, additions to the data base solved the problem. The data base has been rebuilt several times for this reason, and rather than rebuild it piecemeal one more time, we intend to adapt our prograns for analyses of the entire data base rather than selected parts. This will require major changes in our prograns.)

some problems developed involying what can be designated as catchall containers, such as ROT and POT. These are named batches that receive material from many batches, are cleaned out from time to time, and generate MIPs. The batch name, however, does not change. Without some adjustment in our procedures, the generating routines using the audit-trail approach wilj. find one of these catchalls in its family tree and then will branch to all other batches in the data base that also connect to the catchall. There will be many such batches.

Two approaches were used to avoid this difficulty. When using the audit-trail approach, we arbitrarily reassigned the receipt area of all POT and ROT batches to a unique one. This cuts off the trail upon discovering such a lot name and keeps the size of the sets of transactions within bounds. The method, however, requires independent analysis of materials balances for the fictitious receipt areas. This approach was tested and appeared to be working reasonably well, but the discovery of the much simpler algorithm led to the temporary abandonment of the audit-trail algorithm, so this solution to the ROT and POT problem has not been pushed to completion.

When the second algorithm, based on batches, is used to generate MIPs, only POT is a problem and it was tractable from the first. Inclusion of POT led to one rather large MIP, whereas plant technicians had recorded several smaller ones--but our program computed the right sum.

To study the problem all transactions leading to and from POT were printed out in time order along with a running algebraic sum of 
SNM content. The running sum went to zero several times during the year; therefore, we reassigned the names of POT transactions. The first few POT transactions were designated POTA until the sum became zero. Those following were designated POTB until the next zero, and so on through the year. This approach allowed our algorithm to spread the MIP from POT more appropriately over the year rather than including it all at once.

\section{Uncertainty Assignments}

One of our most important tasks was to assign appropriate uncertainties to the MIPs. Heretofore, such uncertainties were only available using hand propagation, an exceedingly tedious and time-consuming task.

Before uncertainties can be propagated, variances must be assigned to each measurement. Data from the measurement control procedures allowed us to determine appropriate systematic and random variances. Becauge PF/LASS only carries SNM amounts to the nearest grari, round-off error is dominant for the balances, the one type of instrument for which we had adequate calibration data. Thus, we adopted an approach of assigning variances by the type of instrument used for a measurement rather than by the specific instrument. The variances were inserted into the data base by the edit routine after reassignment of the measurement code. It was assumed that the variances did not change during the year, that is, recalibration did not occur. Because recalibration did occur, we are overestimating our propagated uncertainties, particularly, as we shall see for Cusum. The formulas used for variance generation are shown in Table VI. Because our task is primarily proof of concept, the assignment of generic rather than specific errors does not invalidate our results. Moreover, because some of the measurement codes are believed to be wrong, a detailed assignment of variances would be pointless.

\section{Negative volumes}

The uncertainties of measurements made with the SAI are known to depend on the plutonium concentration of the sample solution. We should be able to acquire this concentration by eimple aivision of 18 


\author{
Balances \\ Systematic variance $=2.25 \times 10^{-10} \star(\text { SNM })^{2}$ \\ Random variance $=4 \times 10^{-10} \cdot(\mathrm{SNM})^{2}+0.083$ \\ TWC \\ Systematic variance $=6.4 \times 10^{-5} \cdot(\mathrm{SNM})^{2}$ \\ Random variance $=2.9 \times 10^{-3}+(\text { SNM })^{2}+0.083$ \\ TwC (Wet Oxalate Cake) \\ Systematic variance $=64 \times 10^{-6} *(\text { SNM })^{2}$ \\ Random variance $=4.84 \times 10^{-4} *(\mathrm{SNM})^{2}+0.083$ \\ Solution Assay I \\ Systematic variance $=5.6 \times 10^{-5} *(\mathrm{SNM})^{2}$ \\ Random variance $=1.0 \times 10^{-4} *(\text { SNM })^{2}+0.0833$
}

the assayed value and the sample bulk amount; both quantities are reported in the transaction record.

A program was written to list information from transactions having SAI measurement codes. The SNM content, bulk amount, and then the ratio of those two numbers were among those listed. In a significant number of cases the value was reported as negative.

The negative values arise because of an error in the PF/LASS programing that has now been corrected. The error is apparently associated with the nature of a transaction itself. A transaction can be generally defined as the difference between two measurable quantities (rather than the alternative view as the material transferred between two quantities). Thus, when solutions are concentrated, the volumes will decrease and an algorithm based on taking a difference will yield a negative value. This is apparently the root cause of the problem uncovered. 
We have not discovered a procedure for correcting these negative volumes, and we assign an average value for the relative uncertainty of all measurements made with the SAIs.

\section{E. Error Propagation}

The formula used for determining the total variance, $\sigma^{2}$, is

$$
\sigma^{2}=\sum_{i} \sum_{m} \sigma_{\varepsilon i m}^{2}+\sum_{i m}\left\{\sum_{i m} \sigma_{\eta i m}\right\}^{2}
$$

where

$\sigma_{\text {Eim }}^{2}$ is the random error variance assigned to the $m^{\text {th }}$ measurement made with the $i^{\text {th }}$ instrument,

$\sigma_{\text {nim }} 18$ the square root of the systematic error varlance for the $\mathrm{m}^{\text {th }}$ measurement made with the $i^{\text {th }}$ instrument, and

$S_{\text {im }}$ is +1 or -1 depending on whether the particular measurement is added to or subtracted from the materials balance.

The sum is over all measurements. The formulation used here is equivalent to the more usual one and has significant advantages for computation. It is derived in App. B.

A 110-word file was established for both MIP and cUsUM; there were enough words to provide one for each instrument used at TA-55. When a transaction is included in our MIP or CUSUM determination, the appropriate $\sigma_{\eta i m}^{2}$ is added (or subtracted) from the entry in the file corresponding to that instrument. When all transactions have been included, the sum of the squares of these entries is added to the random variance to obtain the total.

\section{F. Editing}

Several instances have been alluded to where editing was needed to assure proper operation of our codes. All of these edits are performed by a single program that edits the entire FFTF data base. The program

(1) assigns corrected values for measurement codes (these are assigned from the batch names in certain cases and from existing measurement codes in others), 
(2) asaigns systematic, random, and total variances according to the prescription of Table VI,

(3) assigns new batch names in place of POT based on when the transaction occurs;

(4) removes the tag placed by the MIP generating routines that flag those transactions used in MIP generation.

The details of this program are given in App. C.

G. Graphical output

Although all our analyses are availabie in printed form, it was essential to develop graphical output. Fortunately, programs for graphical output in the appropriate format were already available* based on the MAPPER ${ }^{4}$ language and were easily adapted for graphing the data shown here.

H. Types of MIP Charts

MIP and CUSUN charts were developed for each of the six FFTF receipt areas (BU, $\infty, P R, O Y, H C$, and $B L$ ) that have MIP accounts (FC does not have an associated MIP account). In addition, a cusum was also prepared for all MIP transactions to. or from any of the FFTF receipt areas. Simllar studies could be made of MIP accounts by pairs (Ref. 5). Besides these normal MIP charts, we also produced MIP and CUSUM charts that ignored MIPs generated in cleanout. This removes the rapid decreases in the cusum charts and makes it easier to spot the steady trends caused by processing alone.

VI. DISCUSSION

A. MIP Charts

Figures 5-10 show the MIPs generated in the FFyF process. They are arranged in the same order as the steps in the process.

The BU unit process shown in Fig. 5 appears well-behaved, although there is some indication that control is worse later rather than early

*These programs were supplied by John Hafer, Los Alamos National Laboratory, Safeguards Systems Group. 
in the year. No single urp exceeds 100 units, making this the "cleanest" process in FFTF by that standard. Also, the average MIP and 1 ts standard deviation is only $-0.50 \pm 19.6$ units. The data appear to break down into two sets: those that are only a few units and those that are several tens of units. The dashed line in the figure is the average MIP, and the dotted lines are two standard deviations to either side. All of the large values involve transactions including scrap or POT, but the converse is not true: most transactions involving POT fall in the small-value category. It is unlikely that the pattern is the result of statistically random variations, so some further examination by the nuclear materials officer (NMO) and process control personnel is probably warranted.

The OD init process is presented in Fig. 6 . The average MIP is $3.9 \pm 53.4$ units. There are several outliers, but most of the negative ones are from cleanout and are, therefore, acceptable. The largest positive point also involves a scrap txansaction.

Figure 7 shows the PR unit process MIPs. The pattern is very similar in shape and magnitude to that of the previous process, OD. The average MIP is $6.64 \pm 58.6$ units. The few outliers are all negative and are assoclated with removing ocrap that had previously been credited to MIP.

The OY unit process is shown in Fig. 8. There is an apparent abrupt change in the distributon of MIP values around batch 55 . This corresponds to a time around mid-June 1980. It is not clear what changed about that time, but process managers and the nko would probably want to study the matter further. The average MIP is $-26.21 \pm$ 84.4 units.

The HC unit process MIPs are presented in Fig. 9. There are two kinds of MIPs: those of only a few units and those of hundreds of units. In almost all cases, the amall MIPs arlse from subdivided lots made from a set of larger batches. Each of these batches (except the last in each series) has a nominal weight of 875 units. The larger MIPs cone from batches with primary lot numbers. Finally, the analler values usually reflect a correction for shipper-receiver differences after chemical analyoie. The largest of the negative points does not 
corrempond to cleanout. It is evident that the average MIP is significantly above zero- $-6.03 \pm 49.3$ units. It is probable that there is a significant measurement bias in this process, probably at the input where the wet oxalate cake from $O Y$ is measured. For accountability it might be useful to separate these two kinds of MIPs into different groups for plotting and analysis, better detecting changes in normality. Any trends in the small MIPs are lost in the scale needed to contain the larger values

Figure 10 shows the MIPs for the BL unit process. Clearly, there is a significant change in the pattern around batch 20. Examination of the details of these MIPs indicates that the first 20 correspond to corrections made to MIP for chemical analysis requested 6 months eariler and, thus, are adjustments to the MIP inventory rather than additions or deletions to it. If we plot the MIP at the time the major transaction activity takes place rather than at the point that the MIP is actually generated, then the first 20 points are assigned to activity from the previous year's campaign. Otherwise, the process seems very well-behaved, the points well described by a statistical distribution with the possible exception of point 50. There is nothing obviously abnormal about that set of transactions. The average MIP is $2.19 \pm 22.2$ units.

\section{B. CUSUy Charts}

Figures 11-22 show pairs of CUSUM plots for each of the six FETF receipt areas. Again, they are arranged in the same order that material moves through the FFTF process. The first of each pair is the plot developed by ignoring transactions from and considering only those to MIP. The "from" MIP transactions are cleanout ones; they are not plus or minus inputs from processing but are changes in the receipt area inventory from cleaning out the box. As we shall see, it is sometimes easier to spot trends in the graphs without cleanout that are not obvious in the totals.

Figures 11 and 12 show the CuSUMs for the BU process. Even when cleanout is neglected (Fig. 11), there are sharp breaks in the generally smooth upward trend. These correspond to the large MIP discussed earlier. The end-of-year cusur of -63 units suggests a measurement 
bias. Incomplete cleanout, to be expected, would leave a positive end-of-year value. Thus, there would appear to be an overall bias in excess of 0.5 units per batch.

Figures 13 and 14 show the CUSUMs for the oD unit process. The first of the pair shows a smooth upward trend with only one statistically significant break around point 45. The MIP causing this break is related to transactions to a scrap category. This smooth trend provides some indication that the process is well-behaved. This is not as apparent in Fig. 14, the second of the OD Cusur plots. Here, the large number of cleanout transactions obscure the smooth trend without indicating the classic saw-tooth pattern that is expected for occasional cleanout. Thus, although the second Cusun chart reflects the accounting of SNM in OD, the first is a useful tool for the NMO or the process manager.

The final Cusun of 874 units plutonium after more than 200 transastions would suggest a measurement bias through this process of less than 4 units. How much less depends on how well the box is cleaned out.

Figures 15 and 9.6 show the CUSUMs for the PR unit process. Again, the former (without cleanout) shows a smoother trend than the latter. The cusus rises rapidly over about 15 batches to about 1000 units, then rises slowly but with what may be slight acceleration. Although the behavior during the first part of the year could be explained by buildup of coatings to saturation, that does not explain the behavisr; subsequently, one would expect level cusur under that assumption. The latter part of the year could probably be adequately fitted by a linear buildup model. Thus, one explanation for the year-long behavior is a two-compartment model for deposit of the MIP--one reaching saturation (such as buildup on the walls of dissolution vessels where some equilibrium might be expected) and one behaving linearly (such as spillage).

The final cusur of 1341 units after cleanout suggests that the measurament bias over the 202 sets of transactions is less than about 6.5 units of plutonium per transaction. 
Figures 17 and 18 show the two sets of Cusur charts for the $O Y$ unit process. Inclusion of cleinout does not noticeably change the shape of the curves but indicates that more than 1000 units of plutonium were recovered through cleanout during the year. The end-of-year balance of 5000 units suggests a very high measurement bias approaching 25 units per batch. We will note below that the next process shows a large bias in the opposite direction, so investigation of a measurement instrument shared by these processes might be instructive. The wet oxalate cake (WOC) thermal neutron colncidence counter (TNC) is a possible candidate.

Figures 19 and 20 show the Cususs for unit process HC. There is little advantage in examining the plot without cleanout except to note that cleanout again provides about 1000 units/year of SNM. The initial downward trend to the data could be explained if an instrument was recalibrated at the end of that time, because there is a corresponding and opposite anomaly in the OY process, a process that has instruments in common with HC. From the HC data we suggest that this hypothetical recalibration must have occurred about the first part of April. The OY data suggest a date several weeks earlier, but the data are not inconsistent with the original date because these first measurements do not use the WOC-TNC. Later measurements may be explained by assuming that the wOC-MNC was out of service until March. A check of the log book bears out this hypothesis.

Figures 21 and 22 show the cusuls for the BL unit process. The curve for BL without cleanout shows 3 distinguishable segments: a smooth gentle rise corresponding to the early corrections for shipperrecelver differences, a second segment also of gentle slope, and then a period of more rapid rise. Figure 22, which includes cleanout, is almost a classic saw-tooth curve. The final balance of 177 units after 81 transactions suggests a bias of under 2 units plutonium per batch.

Figures 23 and 24 show the cusuks for the combined receipt areas. We have simply added all the MIPs together, plotting them at the correct relative times. The combined MIP charts will mean very little, but the custus chart will cancel out internal instrument biases and give a good estimate of how the process is behaving. Because of the large number of points involved, it was necessary to plot the data in 
three separate sections. Examination of the graphs that do not include cleanout show that, except for the early part of the year, the data trend smoothly upward. The first part of the year shows a smooth trend consonant with the second part of the year, but there is apparently anomalous behavior from about batch 25 to 90 . Because there will be a time delay as material moves through the plant, this behavior and correction could be related to the anomalous behavior in the HC or OY process blamed on instrument malfunction. A better way to produce this chart would be to use the audit-trail algorithm starting from output of the last receipt area (BL). The set of receipt areas would be treated as though it were one large recelpt area. Because the execution of that algorithm was not completed, this approach has not been tried.

Incliding cleanout leacs to an acceptable overall process CUSUM of $704 \pm 624$ units and suggests an overall bias of less than 0.6 units per batch. If the number of batches exiting BL is used to count the FFTF process, then the bias rises to about 9 units per batch.

vII. CONCLUSION

The PF/LASS system has provided some very useful information for assessing the accountability of the FFTF process. Although the problems of errant measurement codes and negative volumes are distressing, they are not an absolute deterrent to useful analysis of the data. Some specific recomendations to improve process operations and safeguards follow.

1. Neasurement Biages. Several of the unit processes appear reasonably well-behaved but several have rather significant measurement biases, particularly the HC and OY processes. It is possible that the woc-mic is the greatest source of bias and some effort should be invested in removing such a bias. 
2. POT and ROT. It would greatly assist analysis if the catchall batches such as ROT and POT were renamed after cleanout. Special procechures have to be used to avoid problems caused by this uniform name, and these procedures may be introducing effects that obscure Interesting phenomena.

3. Measurement code Assignments. If exror analysis is to be successful, a more rigorous approach needs to be taken with regard to measurement code assignments. Fither the codes need to be assigned automatically, edited for credibility, or the instruments connected on-line. It is recognized that with the present computer configuration the last is impossible.

4. Round-off. The matter of round-off needs to be examined further. The present procedures reflect a compromise reached many years ago between computer-card size and accountability needs. If a method can be found to maintain more significant figures within the computer while reporting rounded numbers upon output, this would be desirable for error propagation. This step is not necessary, however, until the problem of the measurement codes is solved.

5. Measurement Control. If round-off can be avoided, then the instrument exrors will become more impoxtant and the measurement control procedures will have to be strengthened and followed faithfully.

In summation, PF/LASS is an excellent system providing much highquality data. It continues to be improved by its operators and is a touchstone for other dynamic accounting systems.

\section{REFERENCES}

1. J. J. Malanify and R. C. Bearse, "An Evaluation of the DMAC Demonstration Program (Phase "III Report)," Los Alamos National Laboratory report IA-8953-MS (August 1981). 


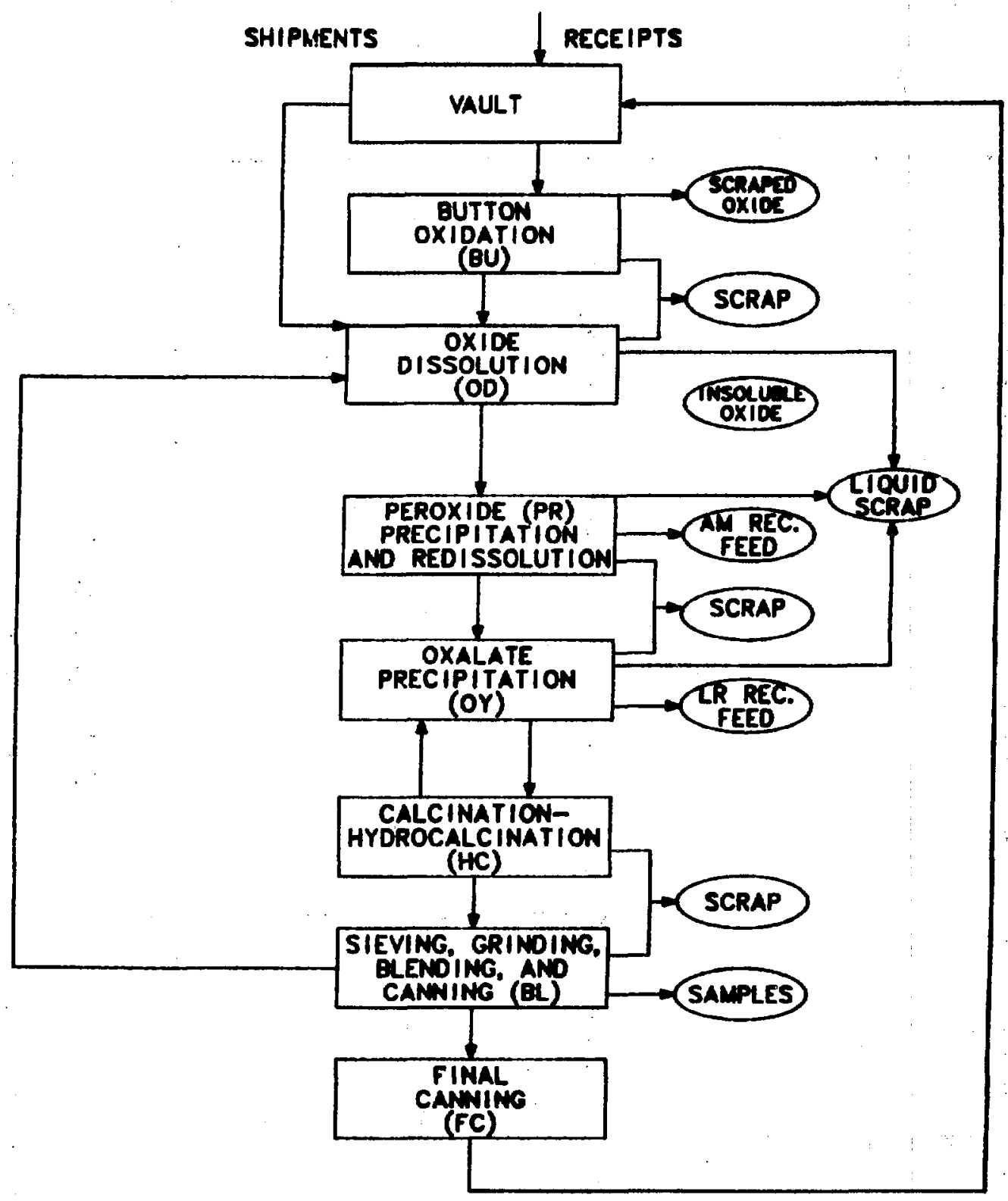

Fig. 1. A aimplified flow chart of the FFTF process adapted from Def. 2 . 


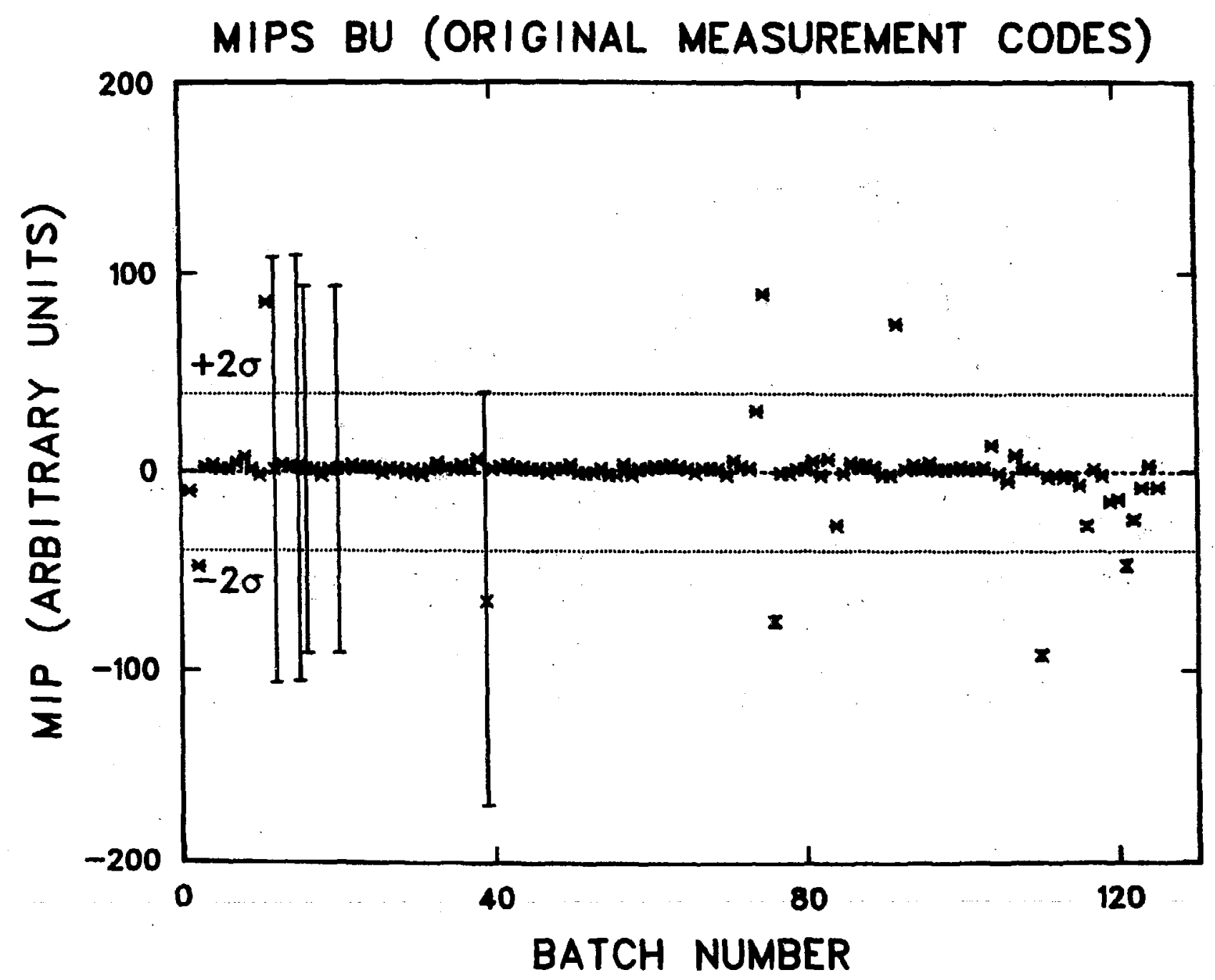

Fig. 2. A plot of the MIPs generated in the BU unit process during the campaign year 1980-1981. Note the five data points with abnormally large error bars, which are believed to result from incorrect measurement code assignments. 


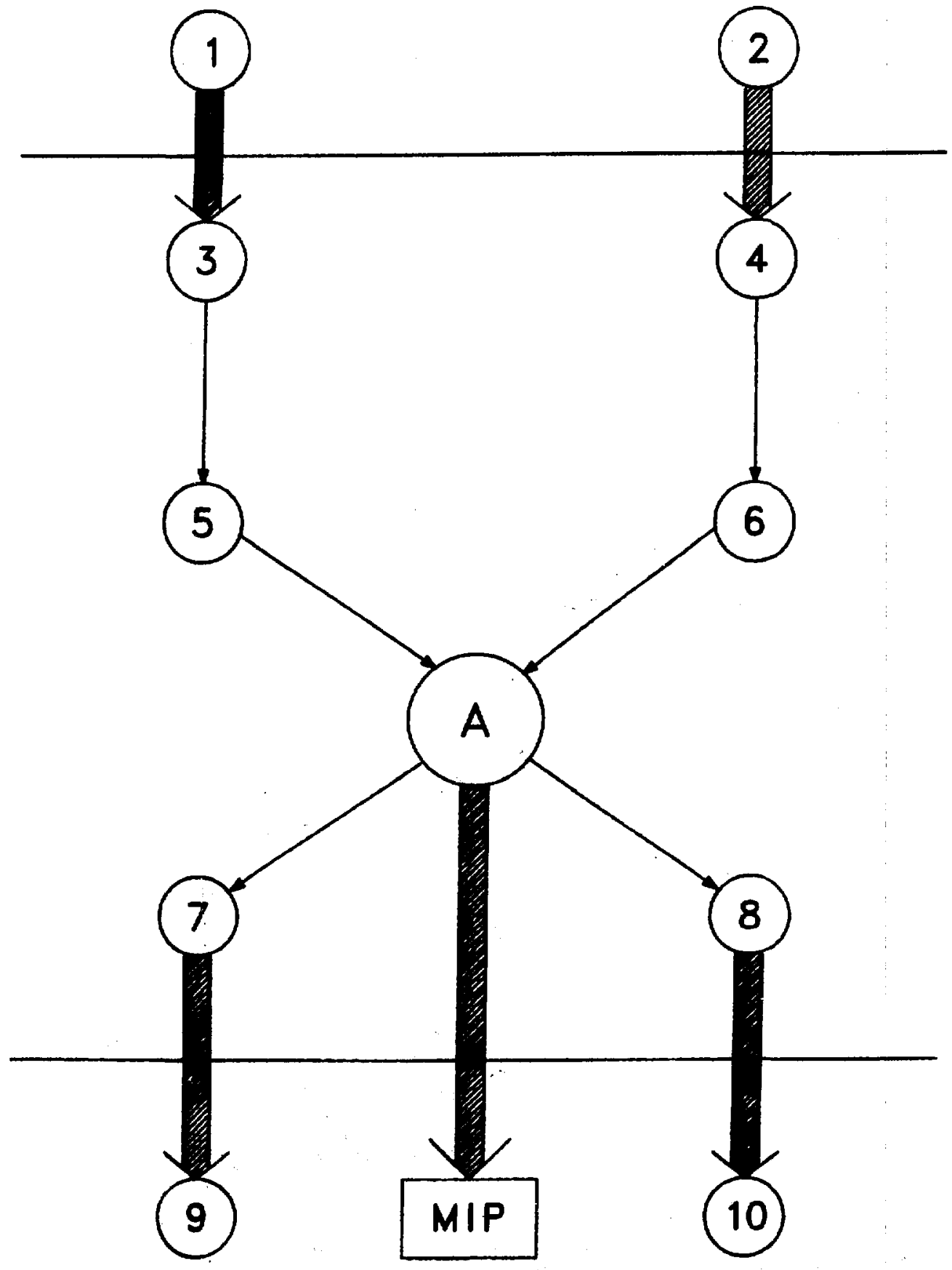

Fig. 3. Conceptual schematic of the processing of batches 1 and 2 into batches 9 and 10 . The recelpt area is represented by the area between the horizontal lines. In MIP can be determined by algebraic sumation of the flowe along the broad arrows that cross the unit process boundary. 


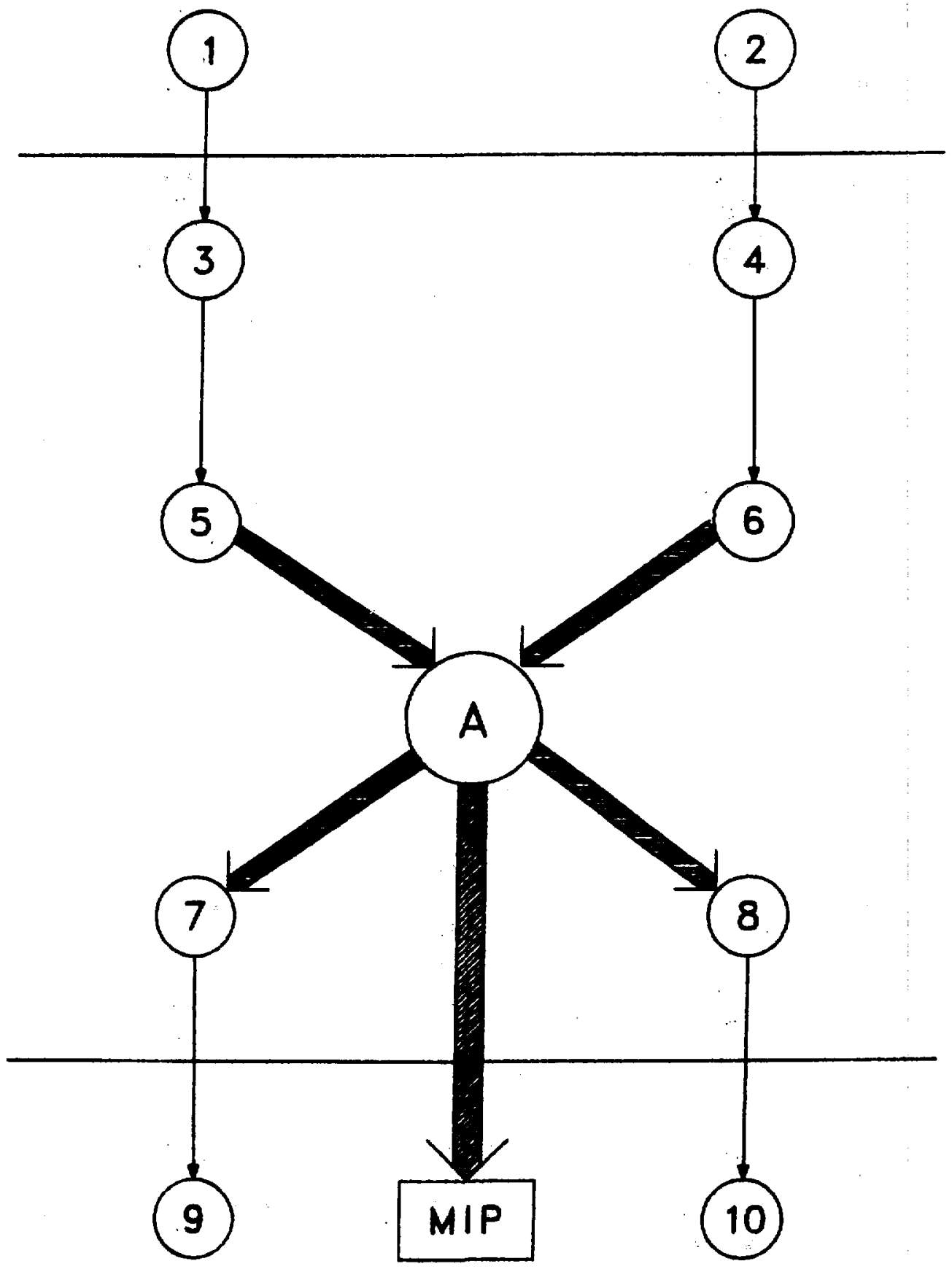

Fig. 4. Alternative to the algorithm of Fig. 3. The broad arrowa represent the transactions to be summed to determine MIP. In this case the transactions considered are those that begin or end in batch $A$. This procedure is used in the present work. 


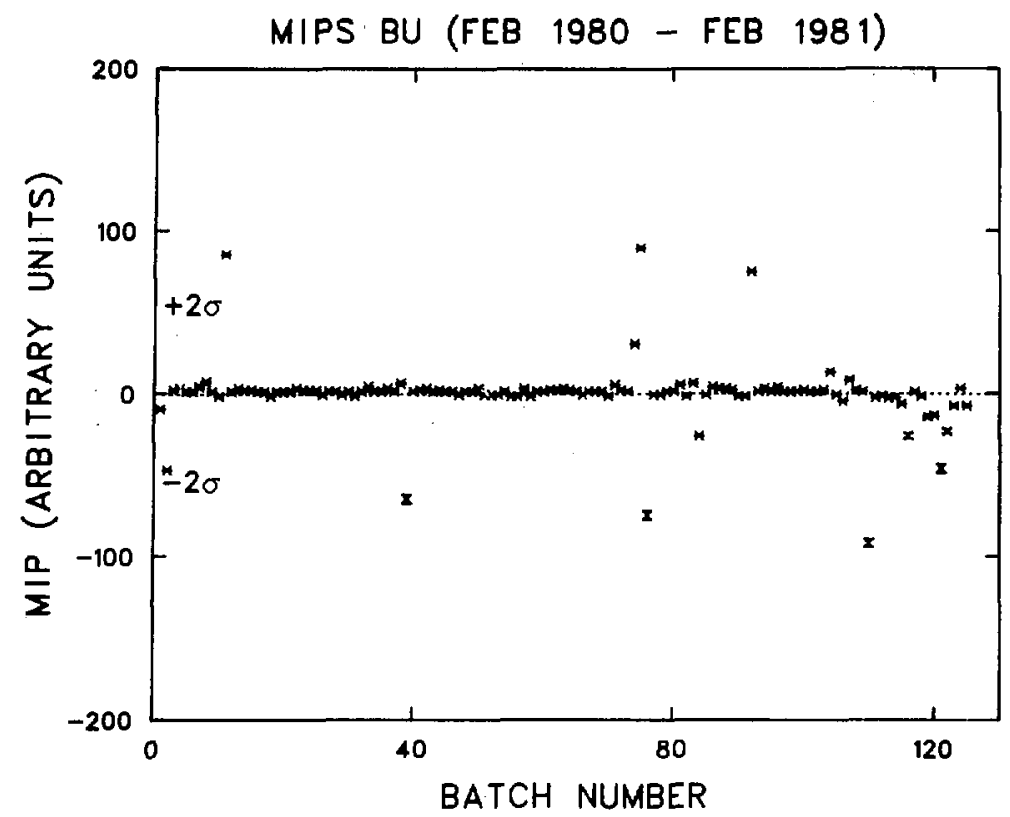

Fig. 5. MIPg from the BU unit process. The dashed line is the average and the two dotted lines represent $\pm 2 \sigma$. Note that the process control is somewhat better during the first half of the campaign year.

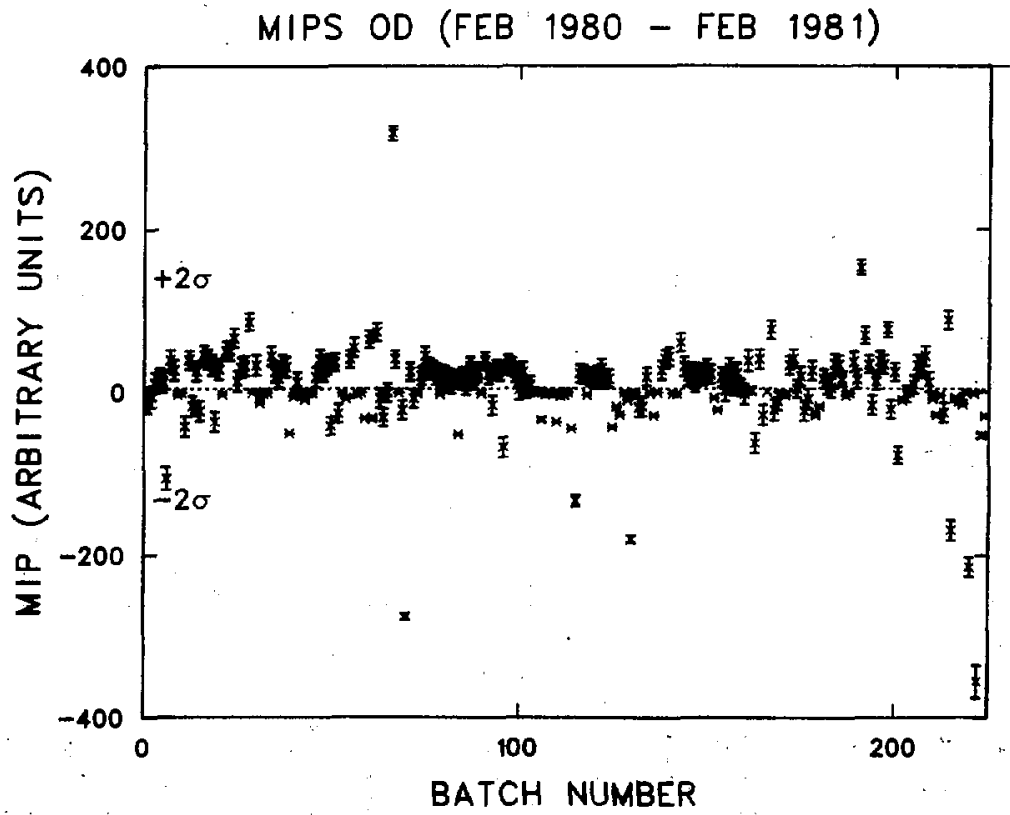

Fig. 6. MIP from the OD unit process. Wote the two different sizes of exror bars. 


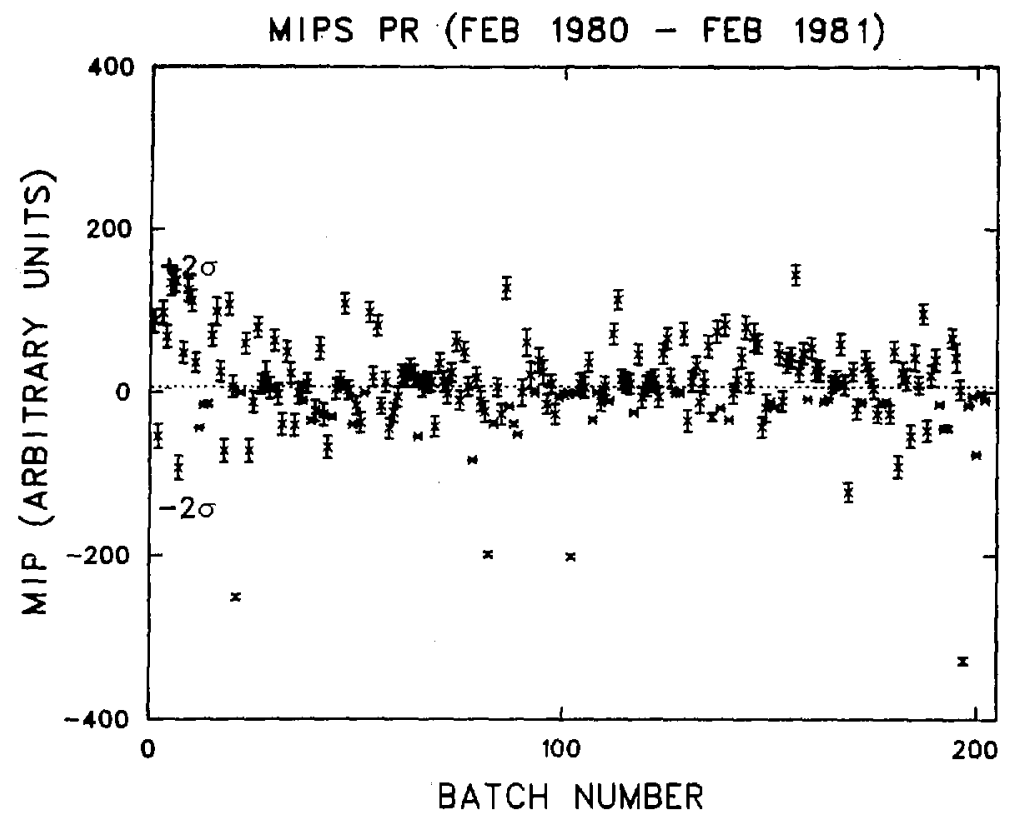

Fig. 7. MIPs from the PR unit process. Note two types of MIPs: those with observable error bars and those with error bars too small to plot at this scale.

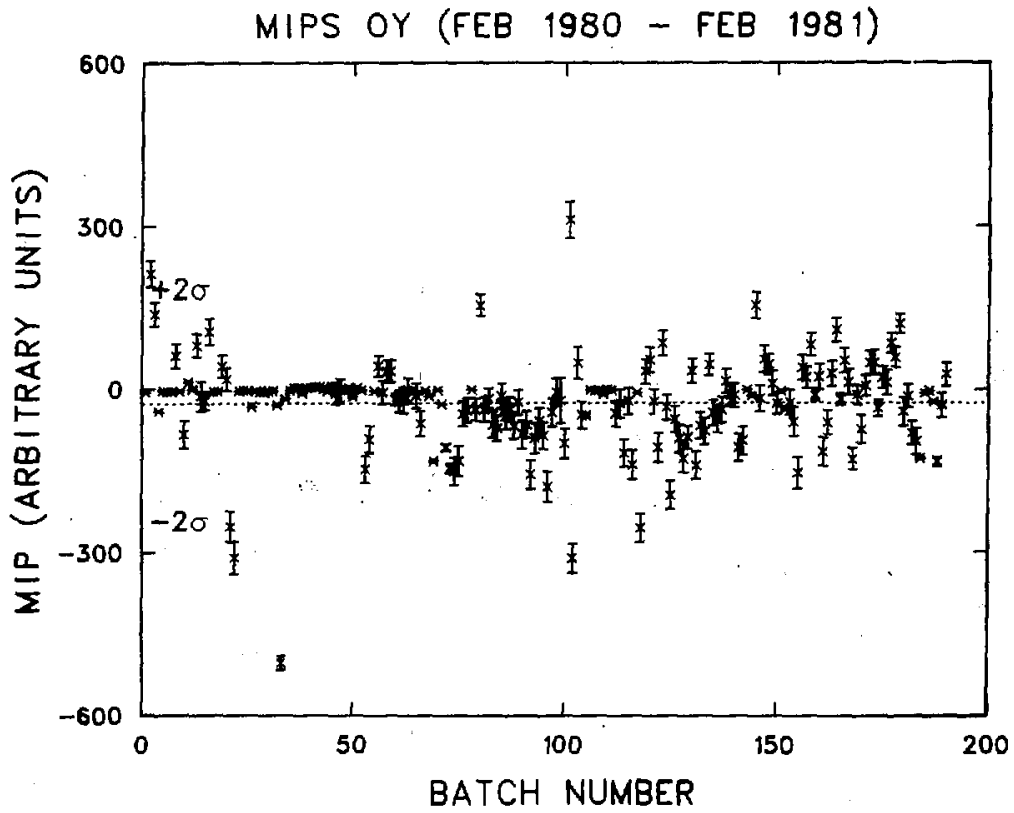

Fig. 8. MIP: from the OY unit process. Again, there appear to be two kinds of measurements: those with a MIP near zero and with very small error bars and those with more dispersion and larger error bars. The former predominate curing the early part of the year. 


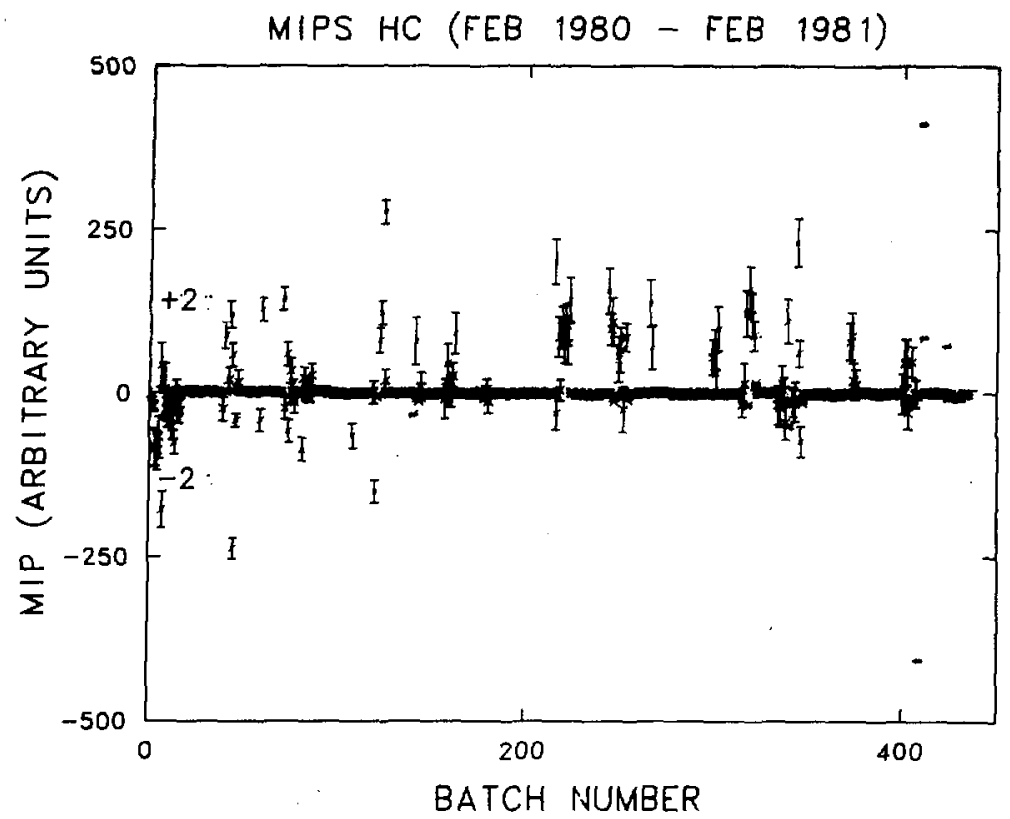

Fig. 9. MIPs fram the HC unit process. Again, there are at least two kinds of data points: those very close to zero with very small errors and those with much larger scatter and larger error bars.

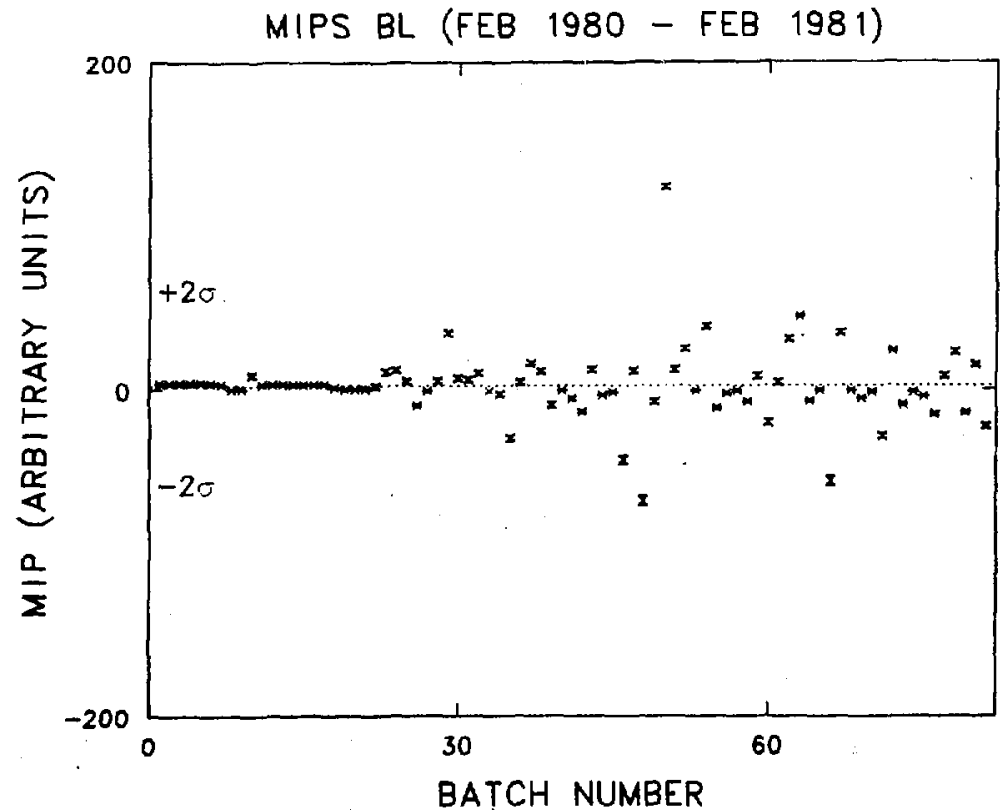

Fig. 10. MIPs from the BL unit process. Note that the scatter of the data appears to increase after batch 25 . The first 25 points cow largely from corrections to the inventory made after receipt of radiochemical analysis and could be justiflably ascribed to the previous campaign year. 


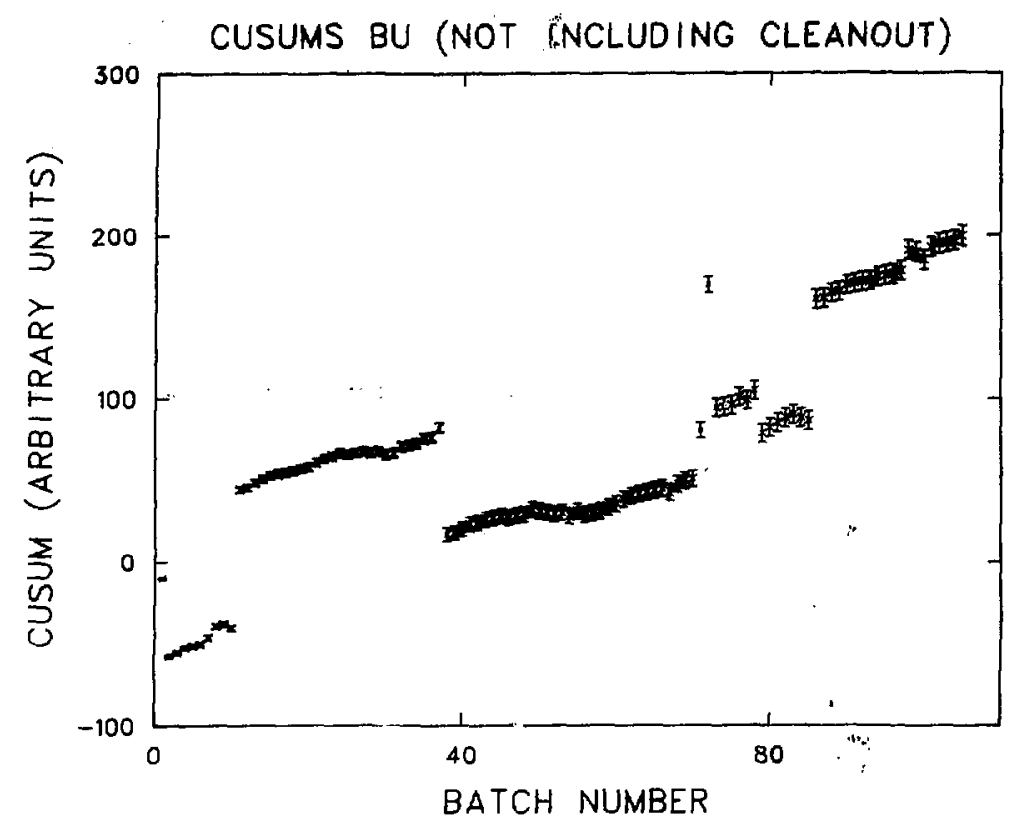

Fig. 11. CuSUMs of the BU unit process with MIPs denoting cleanout included in the total. Thus, the end-of-year drop does not occur and the generally upward trend is enhanced.

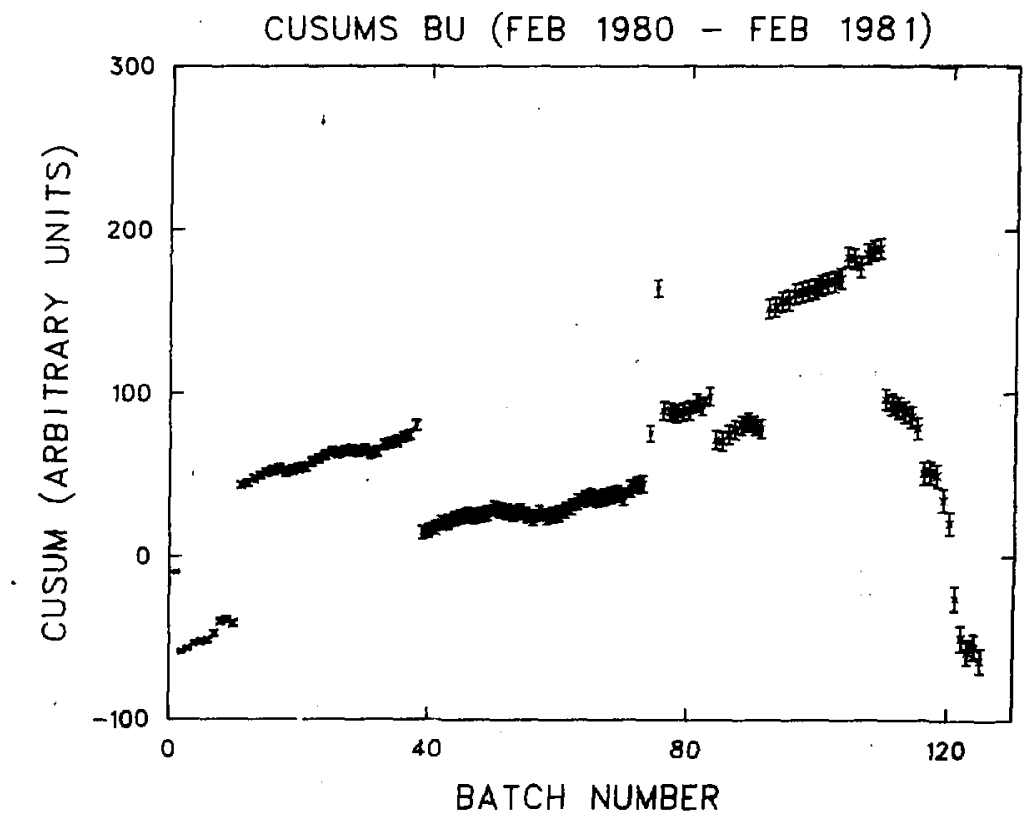

Fig. 12. Cumulative sum of MIP from the BU unit process. Note the significant breaks that correspond to the relatively large MIPs plotted in Fig. 5. The rapid decrease in the curve starting at batch 110 corresponds to end-of-year cleanout. 


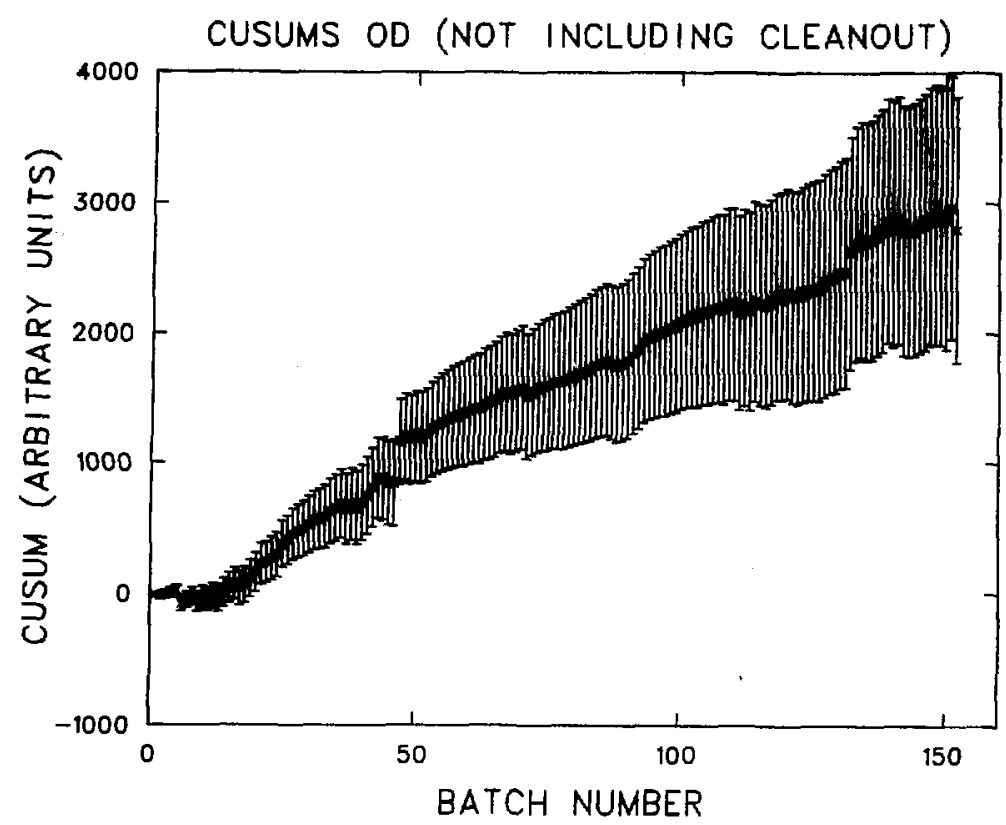

Fig. 13. CUSUMs of the OD unit process, not including MIPs corresponding to cleanout. Here, the generally upward trend is more evident than in Fig. 12 and indicates that the rise in the cusum is due to normal processing effects.

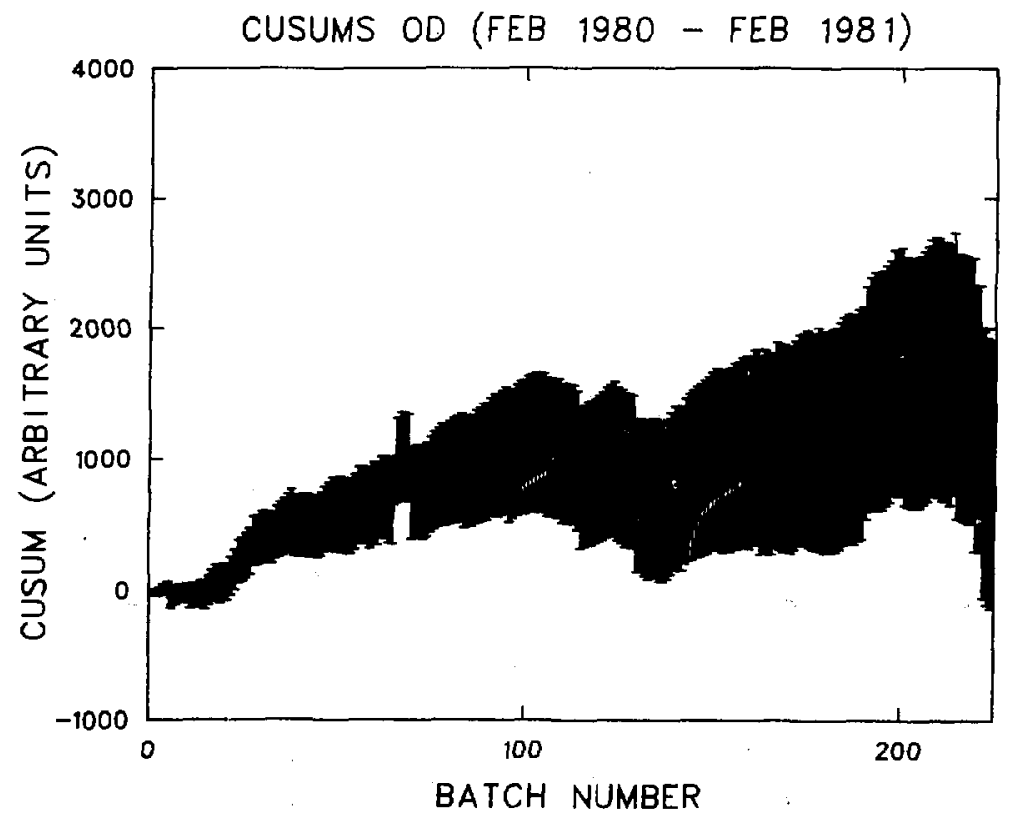

Fig. 14. Custers of the $O D$ unit procesa. Again, end-of-year cleanout is evident starting around batch 220 . 


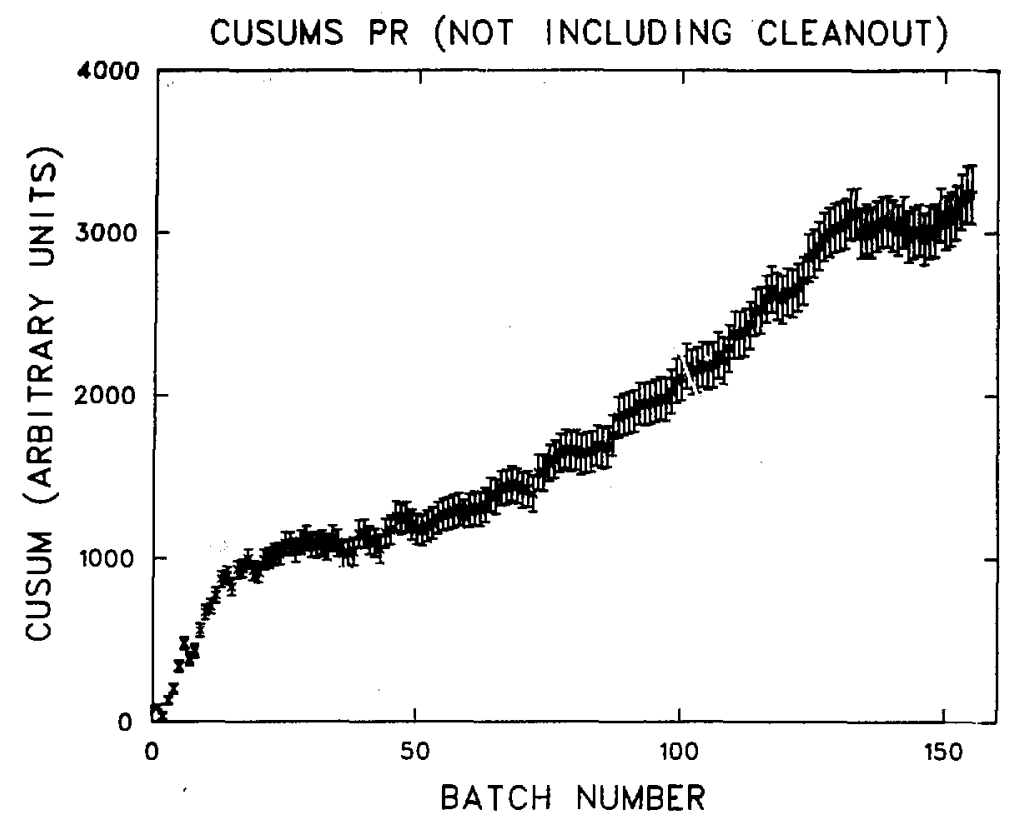

Fig. 15. Cusums for the PR unit process not including cleanout. This plot, neglecting cleanout, can be explained by competing exponential and linear processes for holdup.

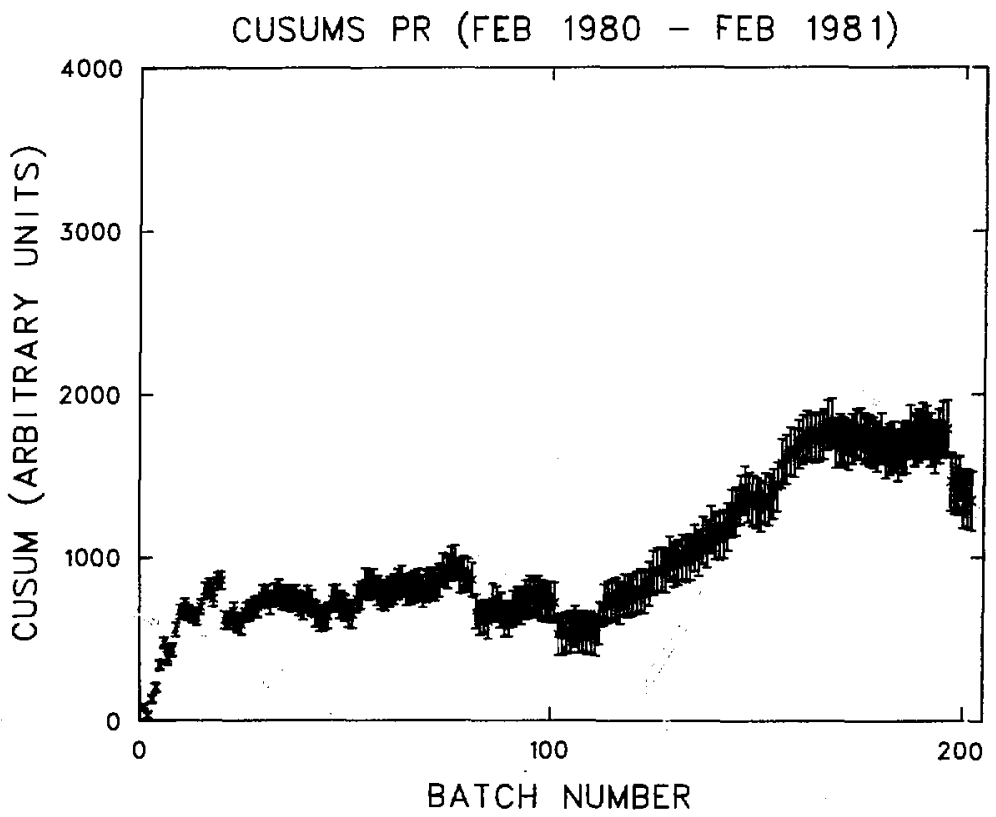

Fig. 16. CUSUns for the PR unit process. The plot is not a classic pattern. It shows a sharp rise early in the campaign year followed by relative constancy. About halfway through the year it trends upward again followed by a second period of constancy before cleanout. This plot should be contrasted with Fig. 15. 


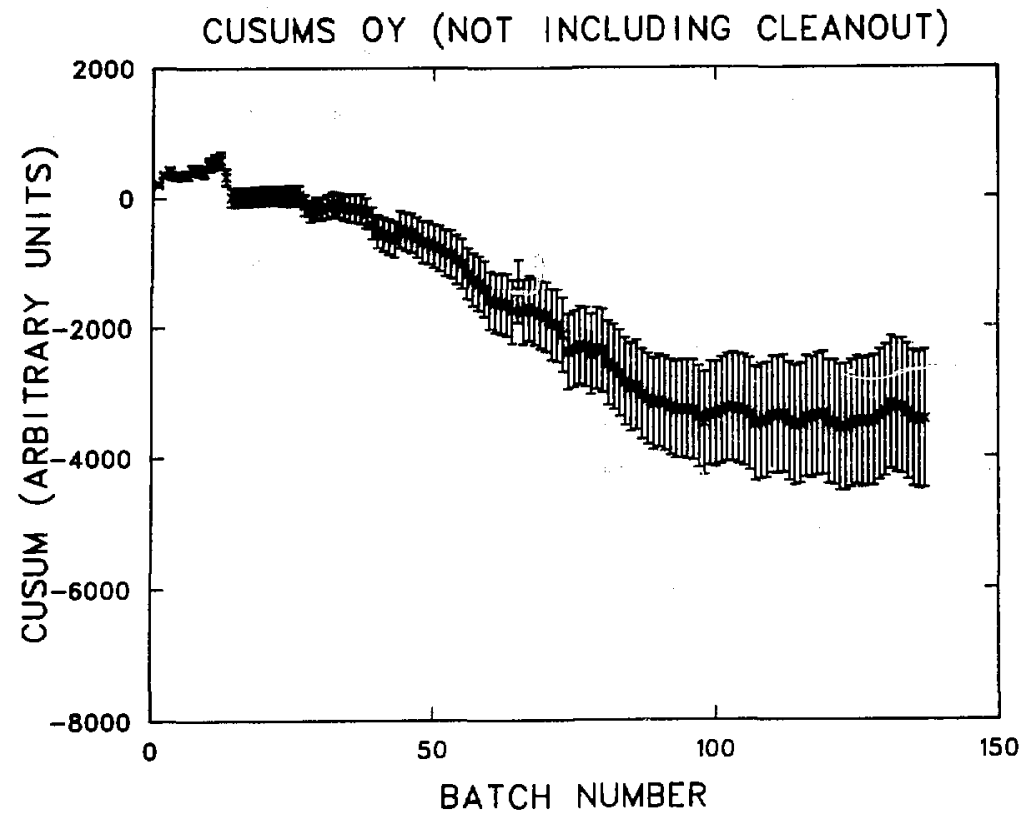

Fig. 17. CUSUMs for the OY unit process neglecting cleanout. A somewhat smoother, more well-behaved curve than shown in Fig. 18 is the result of excluding cleanout.

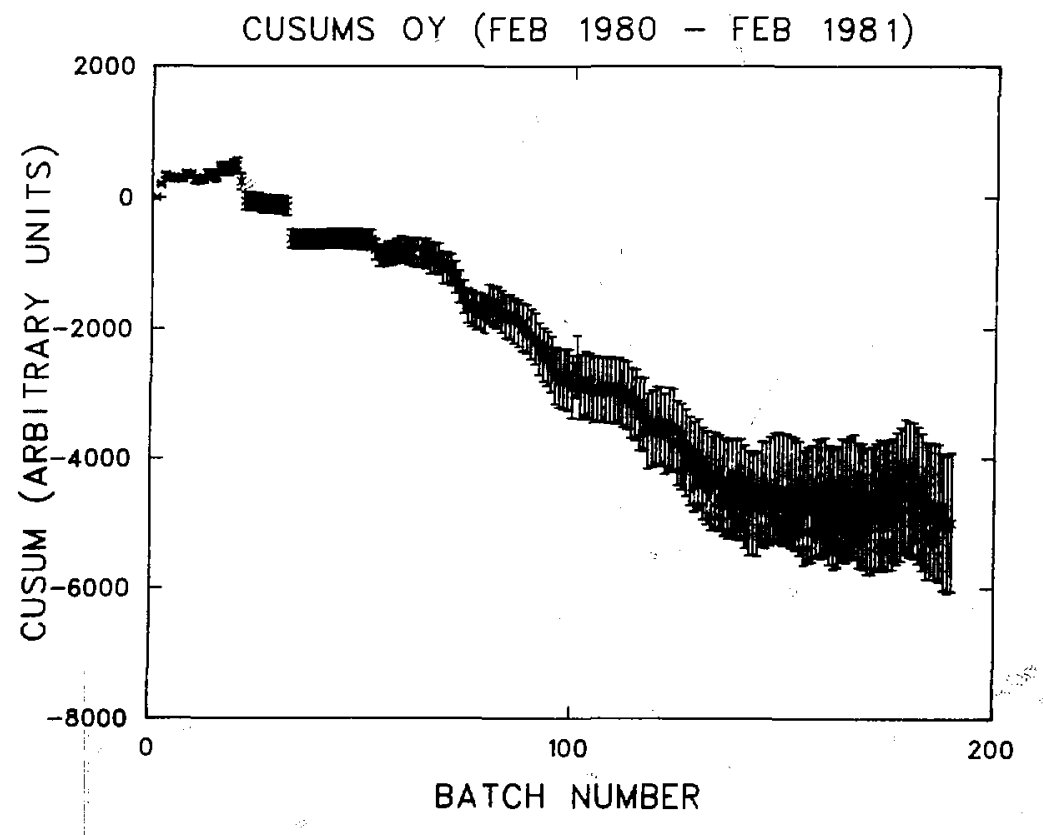

Fig. 18. cususs for the oY unit process. This process has signiilcant negative holdup. This nonphysical effect can most reasonably be explained by bias in one or another of the instruments used to measure, the components of the MIP. 


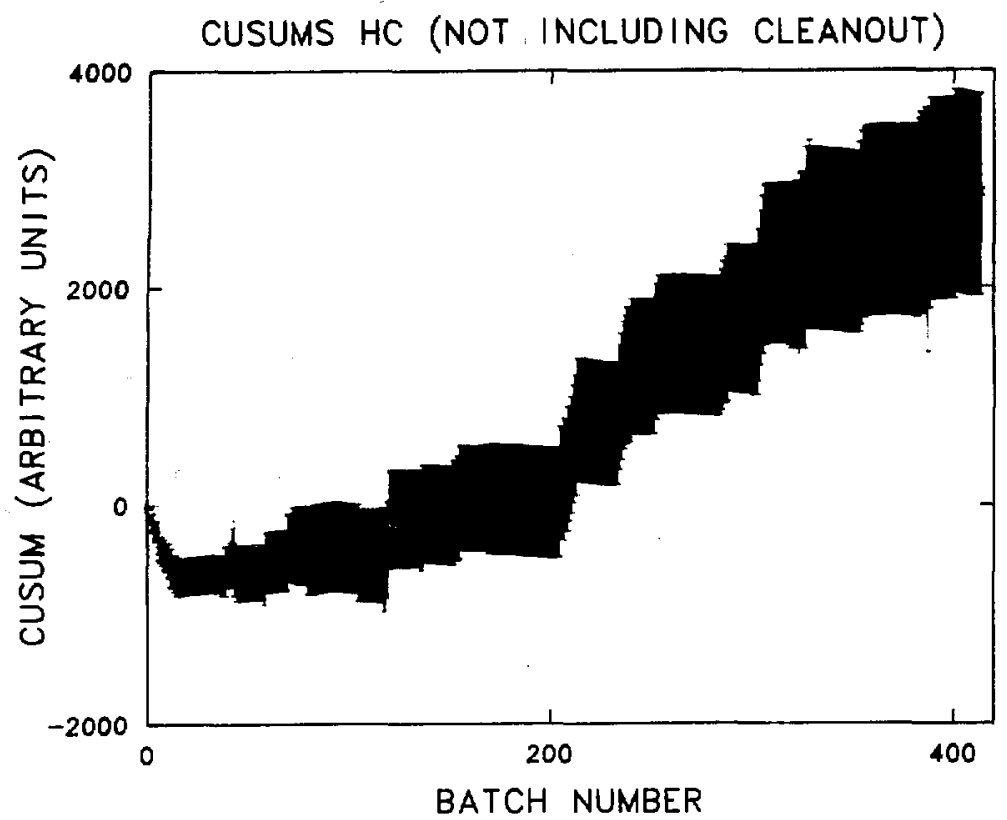

Fig. 19. Cususs for the HC unit process neglecting cleanout. This curve is slightly better behaved than Fig. 20 .

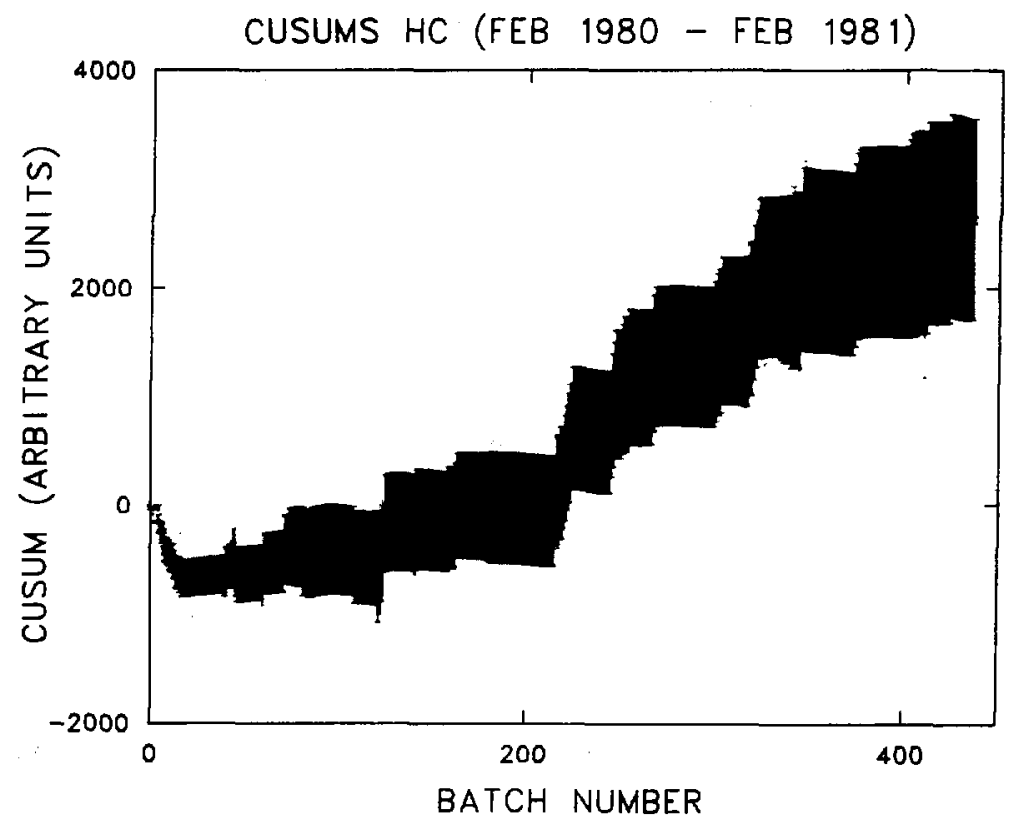

Fig. 20. Cusuis for the HC unit process showing an initial nagatire slope followed by constancy and a final increase. The significant change in the slope early in the campaign year is attributed to a change in the instrument used to measure the MIP components.

40 


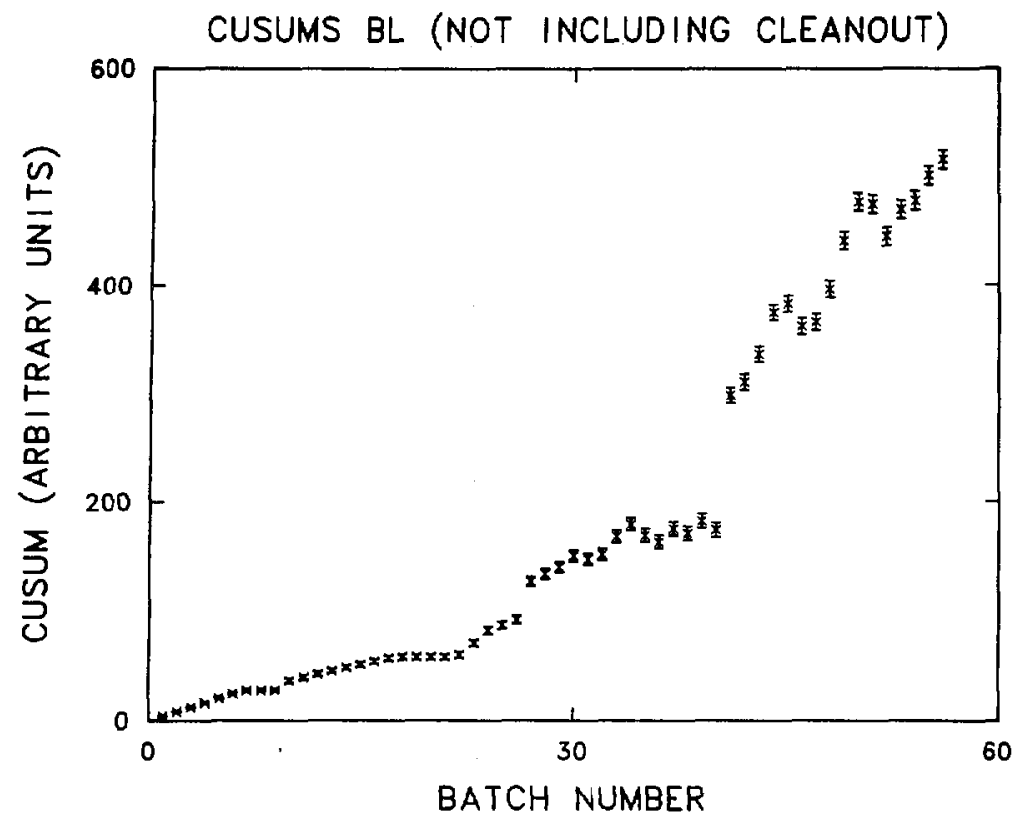

Fig. 21. CusUMs for the BL unit process excluding cleanout. The curve is smoother than in Fig. 22.

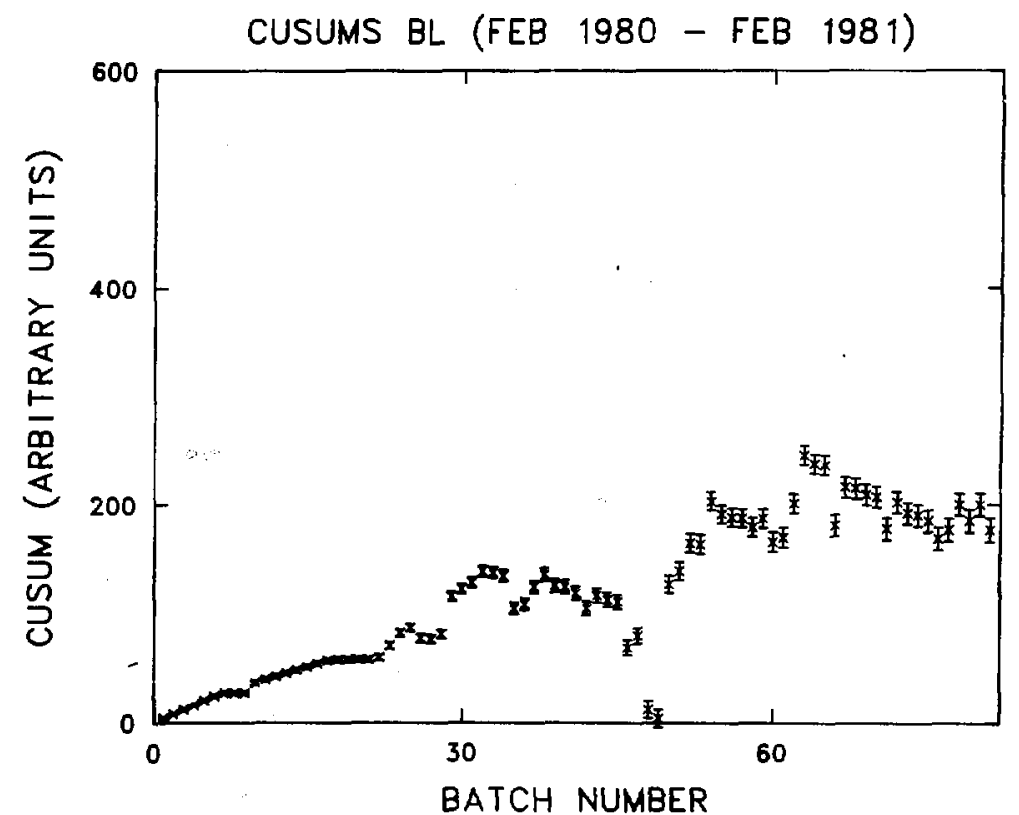

Fig. 22. Cusuls for the BL unit process. This curve comes as close as any to the classic saw-tooth curve that is expected for a process with periodic cleanout. The first $\eta_{20}$ pointa could have been appropriately assigned to the previous campaign year. 


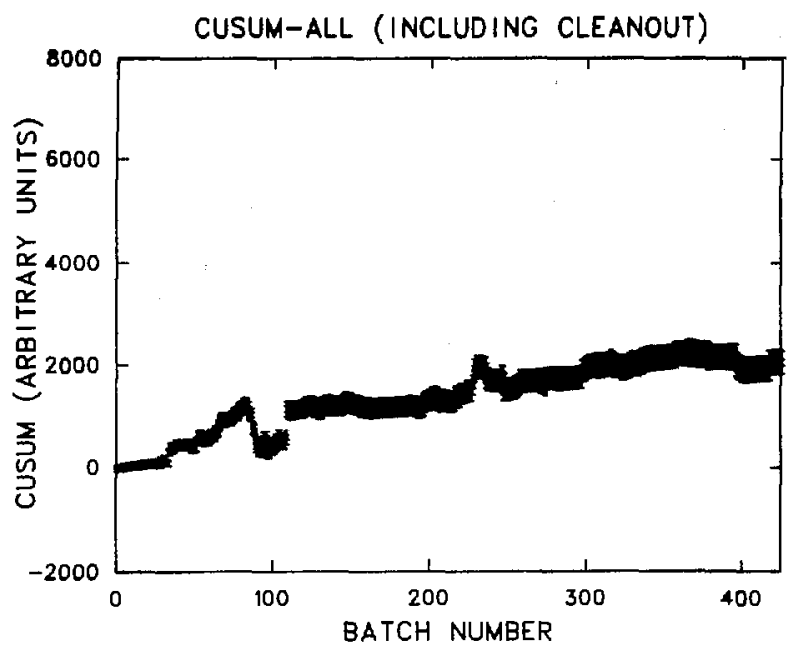

A. Points 0 to 350 .

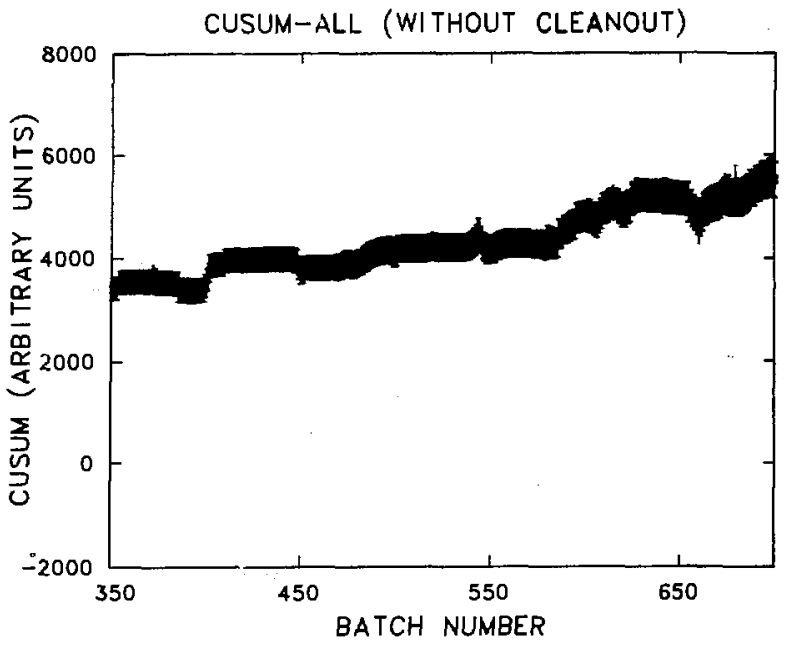

B. Points 350 to 700 .

Fig. 23. CUSUM for all MIPs excluding cleanout. Here, a smooth upward trend after batch 100 is more apparent than in Fig. 24. Comparison to Fig. 24 indicates that 6000 units of plutonium was recovered from cleanout throughout the year. Excluding cleanout decreases the number of points being plotted, but the scale has been expanded so that the year still fills the space allotted.

c. Points 700 to end. 


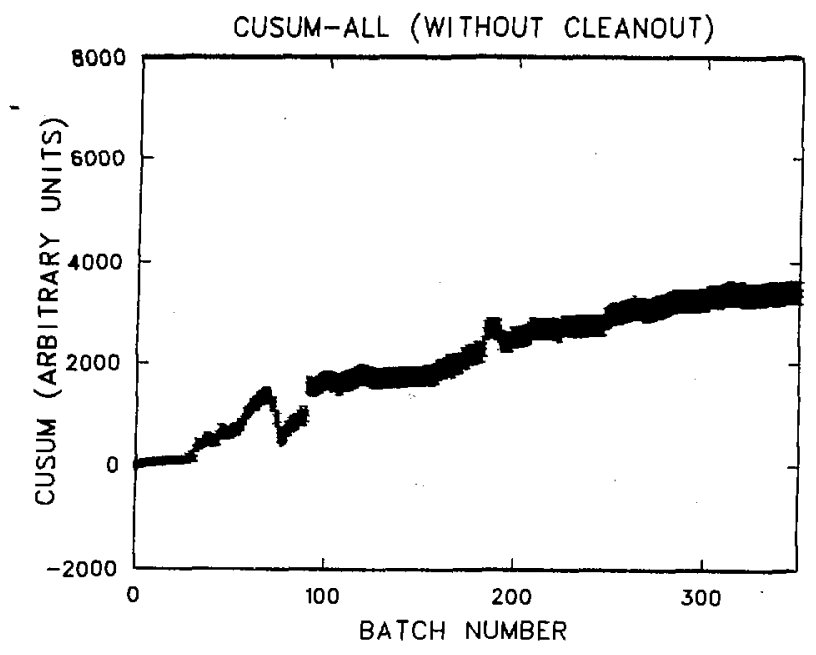

A. Points 0 to 425 .

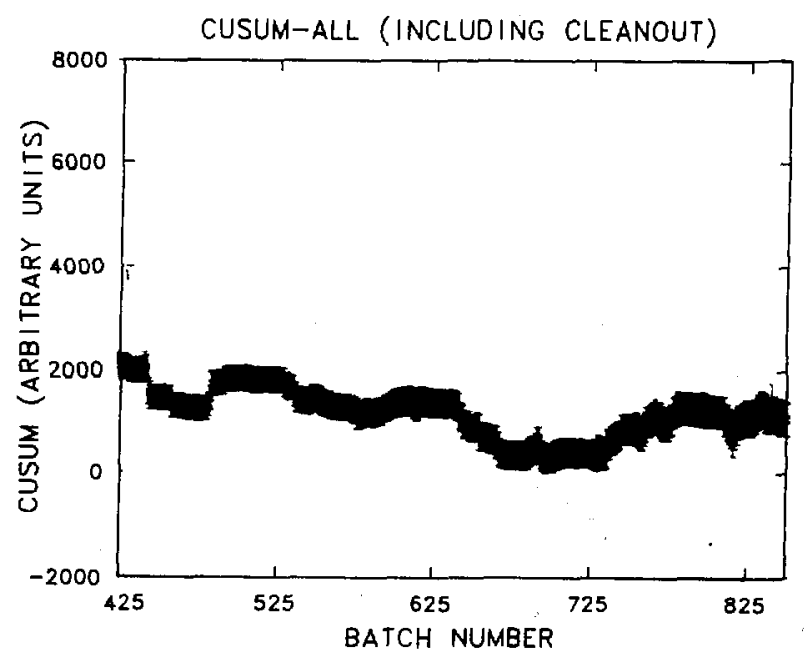

B. Points 425 to 850 .

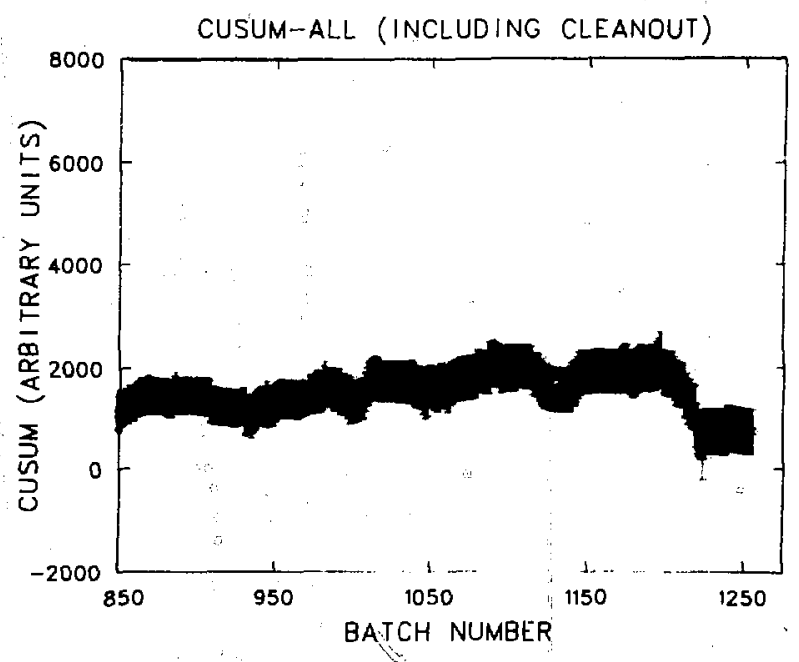

C. Points 850 to end.

Fig. 24. cusur for all MIP* in the FFT procens. The large number of MIPs required that the resulting curve be plotted in three parts. The curve reaches a maximum at $v_{2000}$ units before dropping to an end-of-year value (after clean-out) of 600 units. 
APPENDIX A

TRANSLATION OF DATA

As indicated in Sec. IV, translating the data from Data General Dump Format to a form compatible with the Prime computer was difficult. This process required

(1) decoding the format and then removing the file and record markers unique to Data General,

(2) translating Data General real numbers to Prime real numbers, and

(3) translating Data General alphanumeric representation to that of the Prime.

\section{Decoding the Format}

step I was the most difficult. There are two major types of formats :

(1) that produced when the Data General Computer operated under the RDOS system and

(2) that produced after conversion to the AOS operating system. No information was available to us concerning these formats and, thus, much experimentation was necessary to determine the cipher.

It was finally recognized that the formatting used by the Data General leads to two superimposed patterns of words (in the case of RDOS tapes, bytes). These patterns are shown schematically in Fig. A-1.

For tapes produced before March 1980, the primary pattern was that shown in Fig. A-1A. To remove the record markers we discarded the first word and then alternated between saving 255-word records and disposing of 3-word records until we reached the end-of-file mark on the tape. After the sets of 255-word records were concatenated, the pattern shown in Fig. A-IB was uncovered. To remove the unwanted records, we had to work with bytes (in this case a byte is $1 / 2$ word), not words; this complicated the procedure. We chose to rewrite the entire flle by placing each byte into a separate word in a new file 
(twice the size of the original). From this file we saved the first 18 words (bytes) before beginning a pattern of disposing of 5 words (bytes) and retaining the next 256 . When the retained bytes were reassembled into words, the file was ready for translation.

For tapes produced subsequent to the AOS conversion, the formats are as shown in Figs. A-IC and A-1D. The approach was the same--we stepped through the tape files keepling the material of interest and discarding the unwanted words. Again the second pattern was processed after the first. Fortunately, we worked first with the much simpler AOS format, which 18 entirely word oriented. This gave us the experlence and confidence to persist when the RDOs format proved more difficult to translate.

The Translation of the Real Numbers

An easier problem to solve was that of the difference in internal formatting of real (floating point) numbers between the Data General and the Prime. The two formats were obtained from the respective FORTRAN programing manuals and are shown in Fig. A-2. Unfortunately, we did not have any translated examples of real numbers encoded by the Data General to use in checking our computer codes. We could not be sure of these procedures unt1l the internal evidence fustified it. In fact, our firat version translated positive numbers but returned zero for negative ones. Because negative numbers do not occur in great profusion in the Data Base (most negative numbers occur only in MIP transactions), this fact was undiscovered for several weeks. When corrected, the entire data base had to be rebuilt from scratch--a day's effort.

The easiest problem to solve was conversion of alphanumeric representation. The difference between alphanumeric words in the two ccopilers is only one bit. Thus, a simple routine was written to set bit eight in each byte of alphanumeric, so that the Prime computer would translate the byte properly.

\section{Detaile of Prograns}

Figure A-3 shows a block diagran of the series of prograns that propare the Data General dusp tapes for analyels by the Prime computer. Thewe programe are incorporated into a package called FIxDenNPrs. 
The Data General tapes are read into the Priwe using the Prime utility program MAGNET. After the files are read and the file names made available to EIXDGTXPES, the latter, is executed. REM512, the first program of the series, passes through 1 month's files and removes the file mark that occurs every 513 words. The program Rayov6 then removes the header and the 6 words that occur every 1030 words.

After the file marks have been removed by the two programs above, FIXBIT fixes the alphanumeric words and changes the format for real numbers. In the set of programs for translating earlier data (FIXNICK), this atep is put off until after the selection of FFrF data is made.

SLCTRA selects out those transactions in the translated data base that involve the FFTF process. It does this by keeping any transaction that involves one of the seven PFTF receipt areas (BU, OD, PR, OY, HC, BL, or FC). It also keeps some other transactions that were necessary to close the MIPB. These latter transactions were selected by IOTID nanes determined by trial and error.

Each transaction was written into a separate file with a name of the form FFoxXXXx. He originally intended to have each transaction for the entire data base in its own named file. We discovered during this exercise, however, that the Prime could not efficiently keep track of more than about 1000 files, and thus, although we worked at this stage with many files, the final data base consists of one file.

After each of the transaction files was created, KEYFIL read the date and time in each transaction record and converted that combination into a real number according to the recipe

$$
\begin{aligned}
\text { Time }= & (\text { YR-80) } \cdot 12 \cdot 31 \cdot 24 \cdot 3600+\text { MONTH } \cdot 31 \cdot 24 \cdot 3600 \\
& + \text { DAY } \cdot 24 \cdot 3600+\mathrm{HR} \cdot 3600+\mathrm{MIN} \cdot 60+\mathrm{SEC} \cdot
\end{aligned}
$$

This recipe was chosen so that the ordering of these numbers is equivalent to ordering by date and time. In this way a file of data/time equivalents and their associated record number was produced. This f1le was time-ordered using the program ORDKBY. The order algorithm was a brute force interchange algorithm. The program stops through the file and interchange each paix that is out of order. The program 
is iterated $\mathbf{N}$ times (where $\mathbf{N}$ is the number of entries in the file) to complete the ordering. The ordered file was used to define the order in wich the transactions were added to the file of transactions for the month. The program that accomplished this task was FFisth. This flle, containing a month of data with the transactions now in timeorder and pertaining only to the FFIF process, was then set aside until all the other months had been selected and ordered.

When all the months had been selected and ordered, the program CONCATMATEFPFDATA combined all the data into the final file FFTFDATA. This file was manipulated in the analyses that are presented in this report.

The set of programs FIXNICR were written subsequent to those in FIXDGTAPE. FIXNICK became necessary when the data base for only 1 year proved to be too restricted to correctly compute all MIPs. FIXDGTAPES would not work for these early tapes so FIXNICK was written. The file marks are slightly different, and the subprogram sPLBYT was needed to split the words into bytes because of the byte orientation of the file marks. To speed the translation process it was decided to apply the program FIXBIT only after the FFTF transactions had been selected out rather than before, as was done with FIXDGTAPES. This is possible because, although the alphanumeric formatting between the two computers is different, the Prime "understands" the DG format, even if it uses a different internal format when it writes the same characters. 
RDOS FORMAT

\section{PRIMARY}

A)

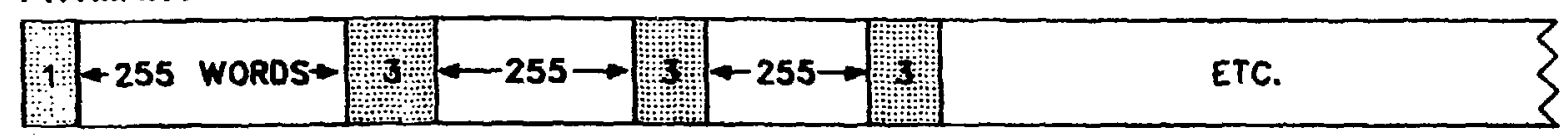

\section{SECONDARY}

B)

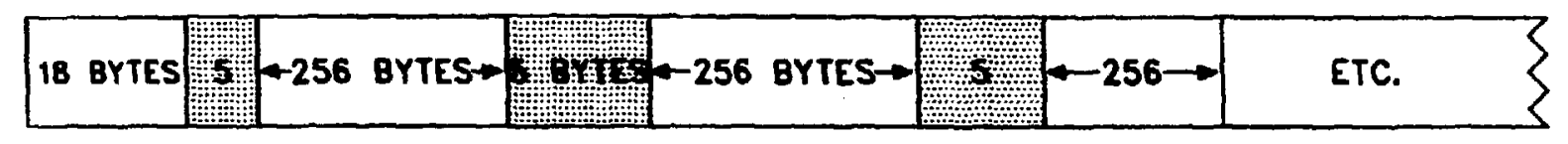

\section{AOS FORMAT}

\section{PRIMARY}

c)

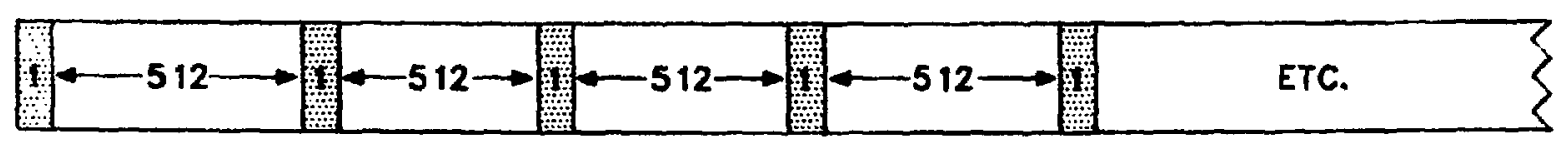

\section{SECONDARY}

D)

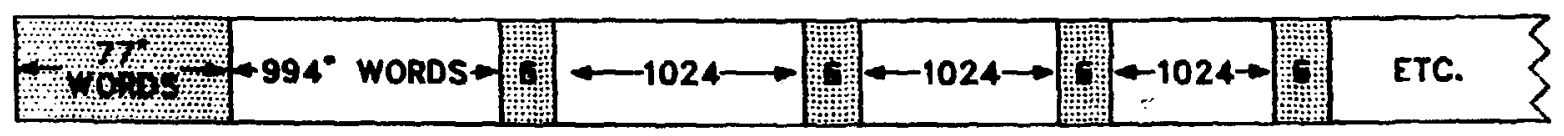

- FOR MARCH \& APRIL THESE beCOME 72 AND 999 RESPECTIVELY

Fig. A-1. File format of the Data General dump tapes that contain the transaction history. $A$ and $B$ show formats produced under the RDOS operating system. C and D show formats produced under the more recent aOS operating system. A new format may be introduced in the near future. 


\section{FLOATING POINT FORMATS}

DATA GENERAL

\begin{tabular}{|l|l|r|}
\hline$\frac{7}{0}$ & EXP & MANTISSA \\
\hline 0 & 1 & \multicolumn{1}{|c|}{78} \\
\hline
\end{tabular}

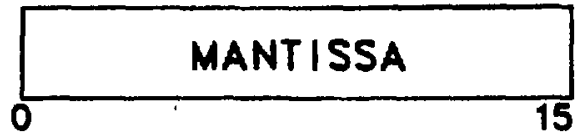

MEXADECIMAL EXCESS 64

VALUE = MANTISSA *16**EX' WHERE EXP' $=$ EXP-6 10

PRIME
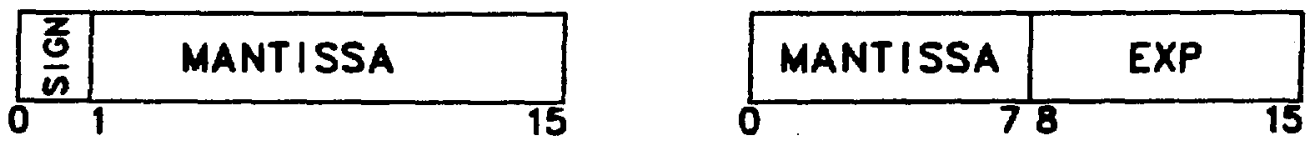

BINARY EXCESS 128

VALUE $=$ MANTISSA * 2*EXP' WHERE EXP' $=$ EXP-128

FOR BOTH

MANTISSA $=\frac{1}{2} B_{1}+\frac{1}{4} B_{2}+\frac{1}{8} B_{3}+\frac{1}{2^{N}} B_{N}$

WHERE N IS THE MANTISSA BIT POSITION

$B_{N}=0$ OR 1; VALUE OF BIT--SET OR NOT SET

Fig. A-2. The bit patterns used in the Data General and Prime computers for representing real-floating-point numbers. 


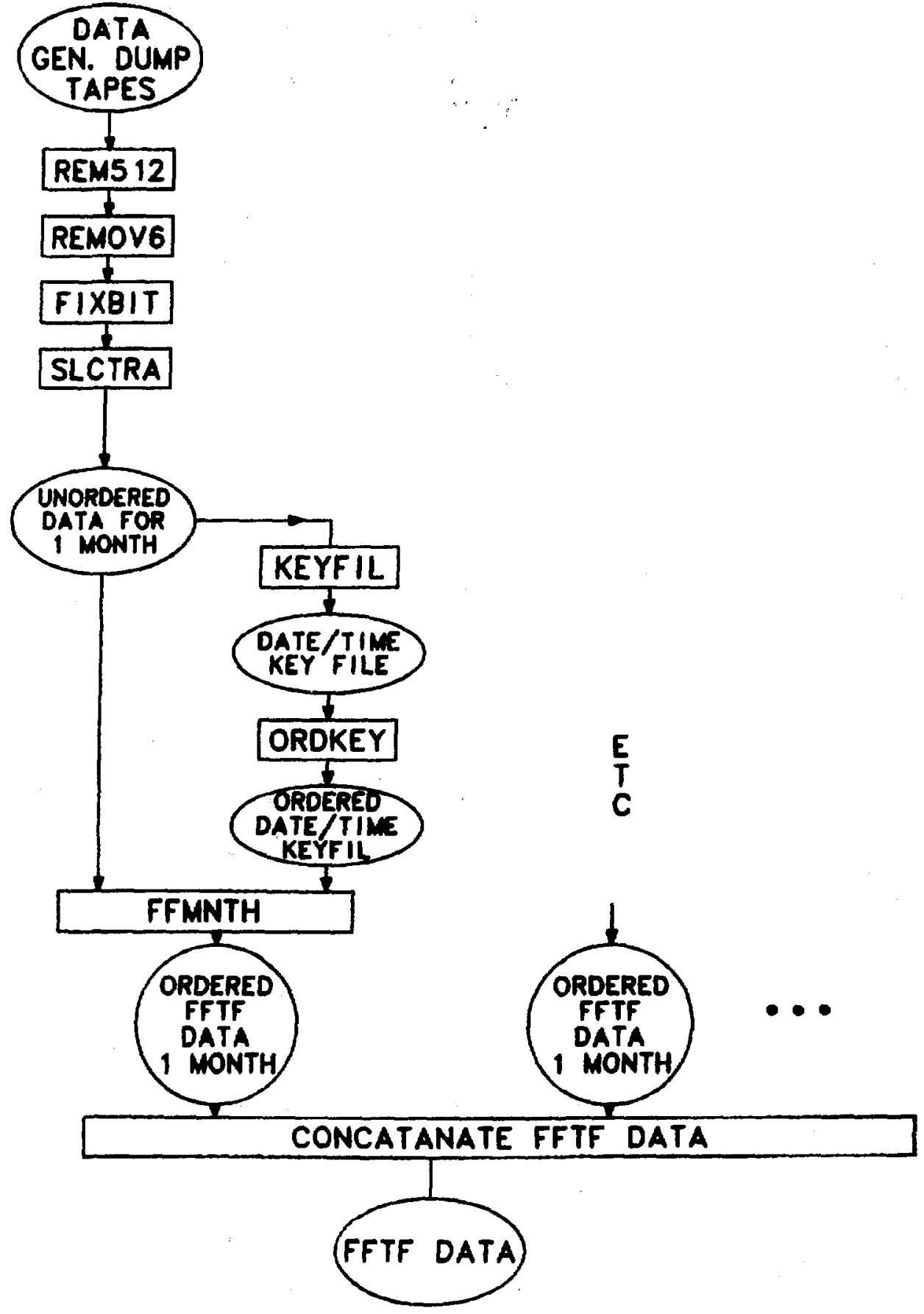

Pig. A-3. Block diagram of the logic used to prepare FFTF data for analysis. Rectangular boxes indicate subprograms and ovals represent data files generated or used by these programs. 
Propagation of errors in the present work took advantage of ${ }^{-}$a variant of the normal propagation relations that was more amenable to computation than is the more standard form. We therefore derive that relation here.

Consider a set of instruments numbered as $1=1,2,3, \ldots, l$. With each of these instruments a series of measurements is made of different samples. The value of each measurement is $x_{\text {im }}$ where $m=1,2, \ldots, n_{i}$ and 1 is, as before, the instrument number.

The random error variance associated with the measurement $x_{\text {im }}$ is taken to be $\sigma_{\varepsilon i m}^{2}$ and the systematic error variance is $\sigma_{\eta j m}^{2}$. We assume that these variances are such that the total variance $\sigma_{i m}^{2}$ may always be written as

$$
\sigma_{i m}^{2}=\sigma_{\varepsilon i m}^{2}+\sigma_{n i m}^{2}
$$

We calculate a materials balance that is the sums and differences of the measurements $x_{\text {im }}$ In general,

$$
M=\sum_{i \text { m }} s_{i m} x_{i m}
$$

where

$$
s_{\text {im }}=+1 \text { or }-1 \text {, }
$$

according to whether $x_{\text {im }}$ is added to or wubtracted from the series to arrive at $M$. 
The usual procedures of error propagation would then lead to

$$
\begin{aligned}
& \sigma_{M, \text { TOTAL }}^{2}=\sum_{1} \sum_{m} \sum_{\varepsilon i m}^{2}+\sum_{1} \sum_{m} \sigma_{\eta i m}^{2}+2 \text { (covariance terms) } \\
& +2 \sum \sum_{i m} \sum_{i^{\prime} \geq 1} \sum_{m^{\prime} \geq m} s_{i m^{\prime}} s_{1 m}, \delta_{1 i^{\prime}} \sigma_{n i m^{\sigma}} \sigma_{11^{\prime} m^{\prime}}
\end{aligned}
$$

where

$\delta_{i 1}$, is the usual kronecker delta for which $\delta_{i 1}=0$ for $i \neq i$ and $\delta_{i 1^{\prime}}=+1$ if $i=I^{\prime}$.

The latter two sums in the last term are restricted in range as shown to prevent double counting. (The quadruple sum is the covarlance térm.)

Because all terms for $i \neq i$ drop out, the equation can be rewritten as

$$
\sigma_{M}^{2}=\sum_{i} \sum_{m} \sigma_{\varepsilon i m}^{2}+\sum \sum \sigma_{\eta i m}^{2}+2 \sum_{i} \sum_{m m^{i}>m} s_{i m} s_{i m}, \sigma_{\eta i m} \sigma_{\eta i m}
$$

Rewriting once more gives

$$
\sigma_{M}^{2}=\sum\left[\sum_{m} \sigma_{\varepsilon i m}^{2}+\left(\sum_{m} \sigma_{n i m}^{2}+2 \sum_{m>m} \sum_{m i m} s_{i m} \cdot \sigma_{n i m} \sigma_{n i m}\right)\right]
$$

Because $\left(s_{i m} s_{i m}\right)$ is either $(-1)^{2}$ or $(+1)^{2}$, we can rewrite the term in square brackets as

$$
\begin{aligned}
& \sigma_{M}^{2}=\sum_{i \mid m}\left\{\sigma_{\varepsilon i \mathrm{~m}}^{2}+\left[\left(\sum_{m} \mathrm{~s}_{i \mathrm{~m}} \sigma_{n, \mathrm{~m}}\right)^{2}\right]\right\} \\
& =\sum_{\lambda 11} \sigma_{k}^{2}+\sum_{i}\left(\sum_{i m} \sigma_{n i m}\right)^{2} \text {. }
\end{aligned}
$$


The first term is now a simple summation of the random variances of all measurements. The second term requires summing (with proper sign) the systematic standard deviations for each measurement made with a particular instrument, squaring the result, and then summing these across all instruments.

This procedure is easily performed with a computer. A memory location is assigned for each instrument and keeps track of $s_{\text {im }} \sigma_{\eta \mathrm{m}}$ as each measurement is considered by the computer. When a materials balance is needed, the contents of each memory location is squared and the result is summed. This is added to the sum of the random error variances (stored elsewhere) and the result is the total variance.

This is the procedure we followed. It works adequately for both MIP and CUSUM. Although we assumed no recalibration took place during the year, such recalibration is equivalent to introducing a new instrument to the collection and could be handled by assigning a new memory location for the instrument after recalibration. 
Figure C-1 shows a block diagram of the programs used to generate the MIPs and CUSUMs.

The FFTFDATA file is generated by the programs discussed in App. A. FFTFDATA is acted upon by several programs to produce the final information and output discussed in this report.

The program EDITFFTFDATA takes the file as prepared by CONCATENATEFFTEDATA and makes changes to allow the other programs to work properly. As indicated in Sec. V.F, it changes measurement codes to what are believed to be more appropriate values and edits the LOTIDS for POT so that all the MIP is not dumped into one transaction.

The edited file is called by SELECTMIPNBW to prepare the list of transactions that involve MIPs. In one version of the program, only cransactions leading to MIPs are recorded. In the other version, transactions arising from or leading to MIPs are included, that is, cleanout is included. This list of transactions is filed in a series of fileg called MIPSLISTXX.

The FFTFDATh file is also used to generate two keyword files for use by other programs. These files consist of the eight-word records MBA/MT/LOTID/RA. (The first digit of MBA is suppressed because it is always "7" for FFTF transactions.) This keyword file can be searched more rapidly than can the full FFTFDATA file.

MIPREAPER searches out the transactions associated with each MIP transaction listed in MIPSLISTXX. It uses FFTFDATA, TBIGIDKEYS, and FBIGIDKEYS to make this selectior. The algorithm is that described in the text and shown in Fig. C-2. MIPREAPER places the set of transactions associated with each MIP in a file MIPTRANSXX.

For each entry in MIPTRANSXX, MIPWRITER obtains the corresponding record from FFrFDATA and somputes the resulting MIP, CUSUM, and associated uncertainties. It produces a formatted output listing to $\mathrm{MIPXX}$ and prepares files MPDATAXX and CMDATAXX for the plotting routines. PLOTYER converts MIPADAXX and CMDATAXX to MPPLOTXX and DMPLOTXC. These latter files are ready for plotting using MAPRER. 


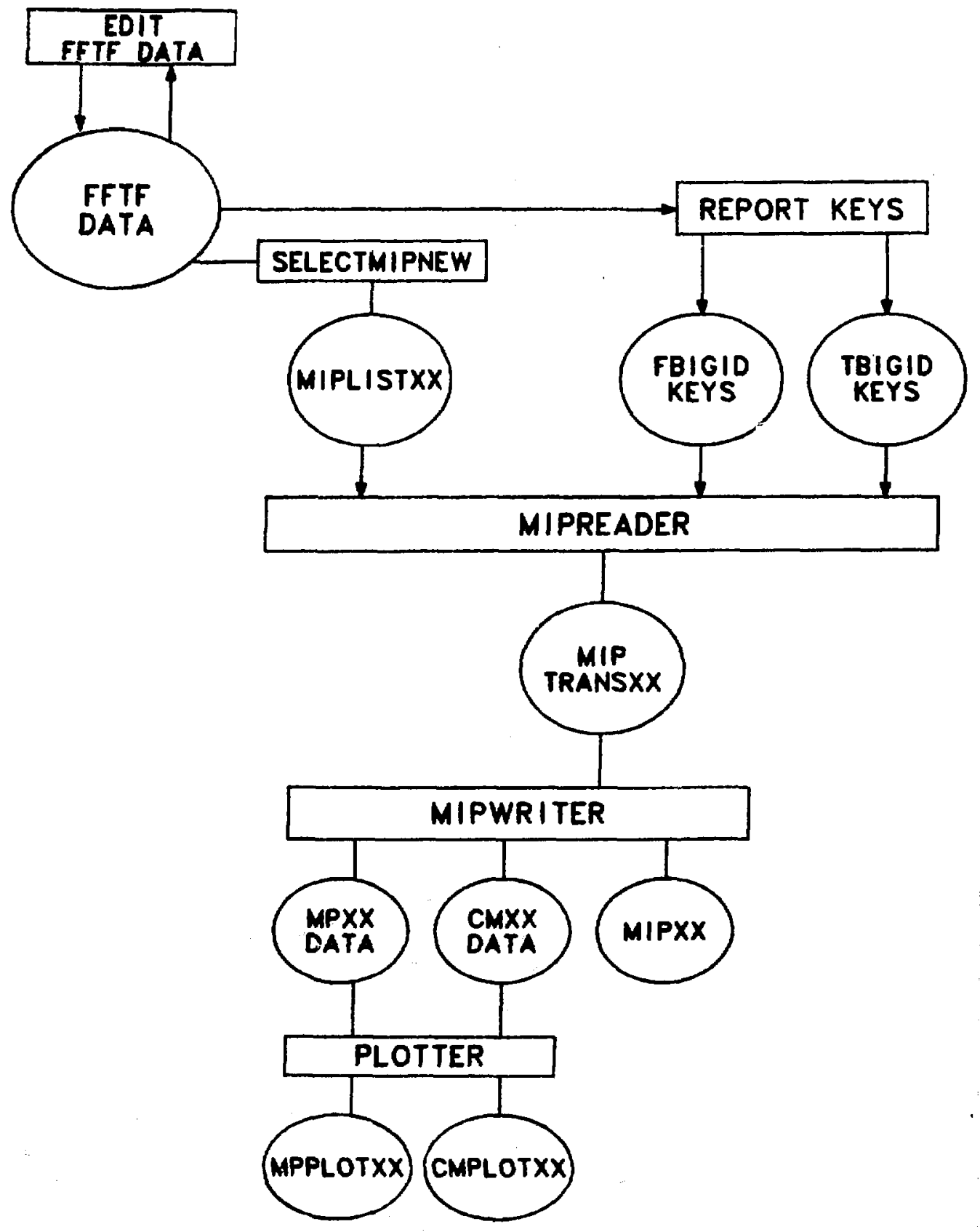

Fig. C-1. Major computer programs and files used to analyze FFTF data. Rectangular boxes represent computer programs and the ovals represent data. 


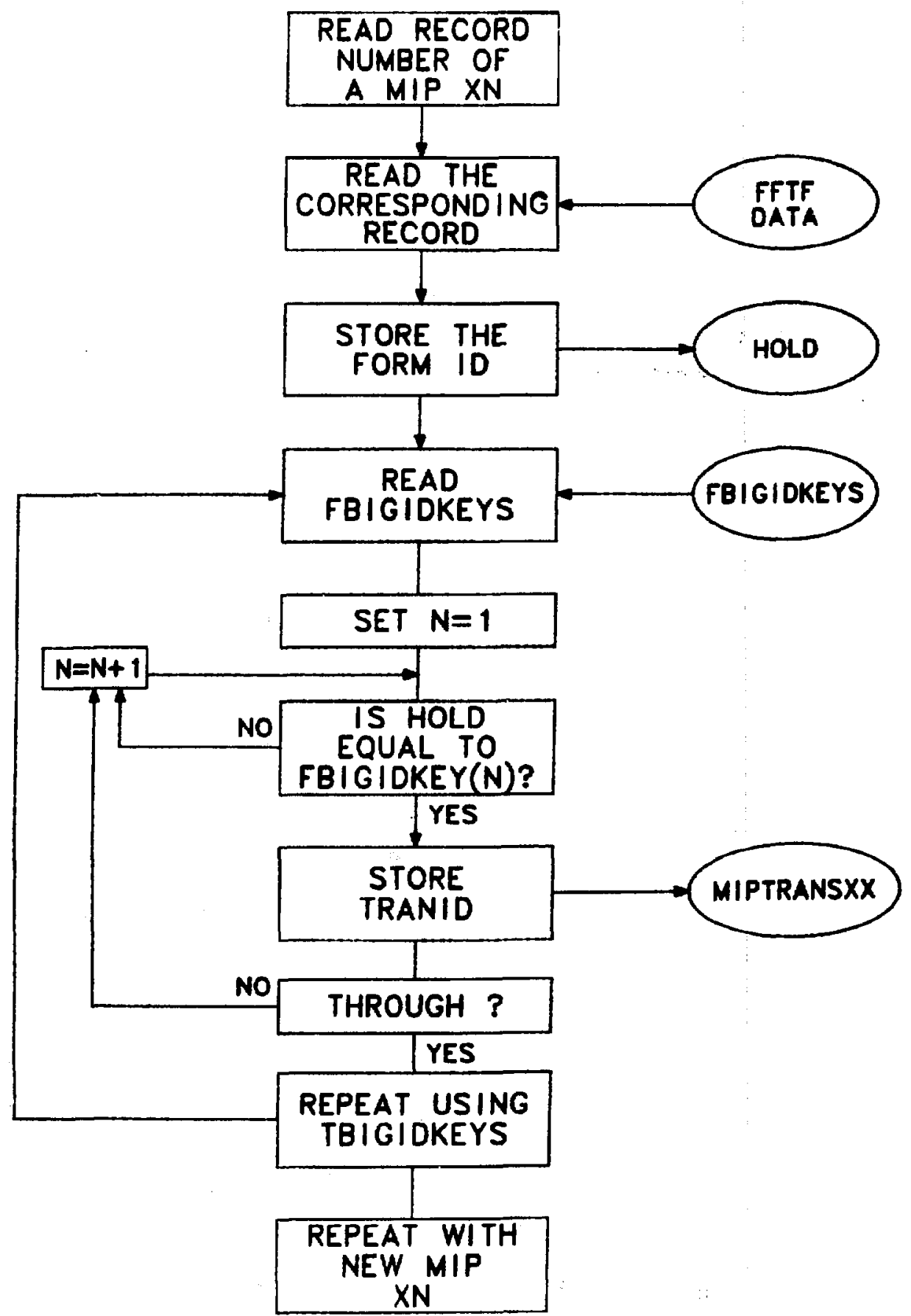

Fig. C-2. The logic used by the program MIPREAPER to determine those transactions corresponding to a particular MIP. The rectangular boxes represent logical steps or operations in the process and the ovals represent the files or memory locations accessed. 